\title{
1 Differential use of multiple genetic sex determination systems in divergent ecomorphs of 2 an African crater lake cichlid
}

3 Hannah Munby ${ }^{1, \dagger}$, Tyler Linderoth ${ }^{1, t^{*},}$, Bettina Fischer ${ }^{1}$, Mingliu $\mathrm{Du}^{1,2,5}$, Grégoire Vernaz ${ }^{1,2,5}$, Alexandra M.

4 Tyers $^{3}$, Benjamin P. Ngatunga ${ }^{4}$, Asilatu Shechonge ${ }^{4}$, Hubert Denise ${ }^{1}$, Shane A. McCarthy ${ }^{1,5}$, Iliana

5 Bista $^{1,2,5}$, Eric A. Miska ${ }^{1,2,5}$, M. Emília Santos ${ }^{6}$, Martin J. Genner ${ }^{7}$, George F. Turner $^{3}$, Richard Durbin ${ }^{1,5,{ }^{*}}$

$6{ }^{1}$ Department of Genetics, University of Cambridge, Cambridge, UK

$7{ }^{2}$ Wellcome/CRUK Gurdon Institute, University of Cambridge, Cambridge, UK

$8{ }^{3}$ School of Natural Sciences, Bangor University, Bangor, UK

$9{ }^{4}$ Tanzania Fisheries Research Institute, Dar es Salaam, Tanzania

$10{ }^{5}$ Wellcome Sanger Institute, Hinxton, Cambridge, UK

$11{ }^{6}$ Department of Zoology, University of Cambridge, Cambridge, UK

$12{ }^{7}$ School of Biological Sciences, University of Bristol, Bristol, UK

$13{ }^{\dagger}$ Authors contributed equally to the work.

14 *Authors for correspondence: t1483@cam.ac.uk, rd109@cam.ac.uk

\section{Abstract}

16 African cichlid fishes not only exhibit remarkably high rates of speciation but also have some of

17 the fastest evolving sex determination systems in vertebrates. However, little is known

18 empirically in cichlids about the genetic mechanisms generating new sex-determining variants,

19 what forces dictate their fate, the demographic scales at which they evolve, and whether they

20 are related to speciation. To address these questions, we looked for sex-associated loci in full

21 genome data from 647 individuals of Astatotilapia calliptera from Lake Masoko, a small isolated

22 crater lake in Tanzania, which contains two distinct ecomorphs of the species. We identified

23 three separate $X Y$ systems on recombining chromosomes. Two $Y$ alleles derive from mutations

24 that increase expression of the gonadal soma-derived factor gene ( $g s d f$ ) on chromosome 7; the

25 first is a tandem duplication of the entire gene observed throughout much of the Lake Malawi

26 haplochromine cichlid radiation to which $A$. calliptera belongs, and the second is a $5 \mathrm{~kb}$ insertion

27 directly upstream of $g s d f$. Both the latter variant and another $700 \mathrm{bp}$ insertion on chromosome

2819 responsible for the third $Y$ allele arose from transposable element insertions. Males

29 belonging to the Masoko deep-water benthic ecomorph are determined exclusively by the gsdf 
duplication, whereas all three $Y$ alleles are used in the Masoko littoral ecomorph, in which they appear to act antagonistically among males with different amounts of benthic admixture. This antagonism in the face of ongoing admixture may be important for sustaining multifactorial sex determination in Lake Masoko. In addition to identifying the molecular basis of three coexisting sex determining alleles, these results demonstrate that genetic interactions between $\mathrm{Y}$ alleles and genetic background can potentially affect fitness and adaptive evolution.

\section{Introduction}

Sex, as a means of generating beneficial combinations of alleles, is one of the most effective evolutionary innovations used among eukaryotes to surmount fitness challenges. Many different means of establishing separate sexes have arisen across the tree of life, operating through a combination of genetic and environmental mechanisms (Bachtrog et al., 2014; Pennell et al., 2018). The continued evolution of new sex determination systems can provide a means to improve fitness via altering sex ratios (Kocher, 2004), resolving sexually antagonistic mutations (van Doorn \& Kirkpatrick, 2007; 2010), and avoiding the negative consequences of sex chromosome degeneration (Blaser et al., 2013). Given this adaptive role of sex determination, this begs the question of whether it is any coincidence that the fastest reported rates of sex chromosome and heterogamety transitions among vertebrates (El Taher et al., 2020) have occurred in East African cichlid fishes, renowned also for their extremely high speciation rates

(Brawand et al., 2014; Ronco et al., 2020). In support of such an association, population genetic models have demonstrated how heterogamety switches arising from a new sex-determining locus coupled with sexual and sex-ratio selection can help generate reproductive isolation in sympatry (Lande et al., 2001).

2 Sex-determination across African cichlid species is largely governed genetically in either a single-locus or polygenic fashion (Ser et al., 2010). The loci controlling sex are known to exist both on homomorphic sex chromosomes, for which there is little if any evidence for long range suppression of recombination around the sex-determining alleles (Parnell \& Streelman, 2013), and on supernumerary B chromosomes (Clark et al., 2017; Clark \& Kocher, 2019). Within the 7 Lake Malawi haplochromine cichlid radiation, the characterized sex determining loci are the orange blotch associated ZW locus and an XY locus on chr5 (Roberts et al., 2009; Ser et al., 2010), two XY loci on chr7 (Albertson, 2002; Parnell \& Streelman, 2013; Roberts et al., 2009), an XY locus on chr3, and a ZW locus on chr20 (Parnell \& Streelman, 2013), using the 
61 chromosome numbering established for the Metriaclima zebra genome (Conte \& Kocher, 2015).

62 In most of these cases, multiple sex determination systems have been observed to act within a

63 single species. Most studies to date have identified sex-associated loci through

64 captive-breeding experiments (e.g. Parnell \& Streelman, 2013; Ser et al., 2010), which provide

65 only broad genomic resolution, or through GWAS on relatively small sample sizes in wild

66 populations with limited power to detect intraspecific associations (El Taher et al., 2020). While

67 these studies point to cichlid sex determination as being highly fluid on the timescale of

68 hundreds of thousands to millions of years, studies on the dynamics within populations would

69 provide the context for examining how recombination, selection, and drift interact with molecular

70 mechanisms to shape the evolution of nascent sex chromosomes (Furman et al., 2020). To this

71 end, we sought to understand how sex determination acts in a single population of the eastern

72 happy cichlid Astatotilapia calliptera.

73 Astatotilapia calliptera is found both in the shallow margins of Lake Malawi as well as in the

74 surrounding rivers and smaller lakes. Peterson et al. (2017) found that the major chr7 XY locus

75 previously identified in Malawi Mbuna cichlids determined sex in a population of $A$. calliptera

76 from Lake Malawi. Despite only mapping the effect to megabase-scale resolution, they

77 postulated that a variant in the gonadal soma-derived factor ( $g s d f$ gene on chromosome 7 was

78 responsible for dictating sex given its repeated role in sex determination in other fish species

79 (Einfeldt et al., 2021; Jiang et al., 2016; Kaneko et al., 2015; Myosho et al., 2012).

80 In particular, we studied A. calliptera in crater Lake Masoko to the north of Lake Malawi, which is

81 estimated to have formed $\sim 50,000$ years ago (Williamson et al., 1999). Lake Masoko is only 700

82 metres in diameter with a shallow littoral margin and walls steeply descending to around $36 \mathrm{~m}$ at

83 its deepest point (Turner et al., 2019). It is currently a closed system, without surface

84 connections to any other water bodies (Turner et al., 2019). With the only other fish being two

85 cichlid species distantly related to $A$. calliptera and one clariid catfish species, the lake provides

86 a relatively simple context for studying the evolutionary genetics of sex determination,

87 speciation and their potential interaction. Genomic evidence suggests that $A$. calliptera

88 colonised the shallow littoral habitat from nearby river systems $\sim 10,000$ years ago, and

89 subsequently extended its range into the deeper benthic habitat $\sim 1,000$ years ago (Malinsky et

90 al., 2015). These shallow littoral and deep benthic populations are phenotypically distinct

91 ecomorphs, with the differences in habitat use coinciding with differences in body shape and jaw

92 morphology. Moreover, the ecomorphs can be distinguished by differences in male breeding 
93 colouration, with reproductively active littoral males being typically yellow, and benthic males

94 dark blue. Both ecomorphs are sexually dimorphic, with males generally larger and more

95 brightly coloured than the females, which tend to have a duller, silvery brown colouration.

\section{Results}

97 We collected whole genome shotgun sequencing data for 548 Astatotilapia calliptera from Lake

98 Masoko at a median coverage of $14.5 x$ (range $4.5 x-22 x$, mean of $12.2 x$ ), and combined this

99 with data from 99 previously published samples (Malinsky et al., 2015), resulting in whole

100 genome sequence data for 596 male and 51 female fish (Supplementary Table 1). European

101 Nucleotide Archive accessions for the raw Lake Masoko A. calliptera sequence data are

102 provided in Supplementary Table 1. Reads were mapped to the high-quality fAstCal1.2 A.

103 calliptera reference genome and variants called at 3,328,052 quality-screened single nucleotide

104 polymorphism (SNP) sites (see Methods for details). All commands, code, and links to

105 downloadable source data used to generate the following results and figures can be found at

106 https://github.com/tplinderoth/cichlids/tree/master/Masoko_sex_study.

\section{Multiple $Y$ alleles determine sex in Lake Masoko}

108 We carried out a genome wide association study (GWAS) for sex using a linear mixed model

109 framework (Figure 1a). The most strongly associated SNP is very highly significant $\left(\log _{10}\right.$

110 p-value $=2.02 \mathrm{e}-22$ ), and located at position 18,098,212 on chromosome 7 approximately $8 \mathrm{~kb}$

111 downstream of the gene gsdf. By considering read depth summed over all fish heterozygous for

112 this SNP, we established that it, and the entire gsdf gene, are contained in a $20 \mathrm{~kb}$-long region

113 that exhibits 50\% inflated relative coverage in the heterozygotes, suggesting that the associated

114 variant chromosome contains a duplication of this region (Figure 1b). We examined paired end

115 Illumina reads from Masoko A. calliptera samples homozygous for the apparent duplication

116 (Supplementary Figure 1a), and long Pacific Biosciences reads from a male fish from a related

117 species (Tropheops sp. 'mauve') which also shows the inflated coverage pattern

118 (Supplementary Figure 1b), and in both cases confirmed the presence of a tandem duplication

119 spanning coordinates $18,079,155$ to $18,100,834$ of chr7. We also confirmed the presence of this

120 duplication junction by PCR (Supplementary Figure 1c). Copy number of the duplication is a

121 stronger predictor of sex than the best associated SNP from the GWAS scan (Table 1),

122 suggesting that the duplication itself operates as a $Y$ allele in an $X Y$ sex determination system. 
(a)

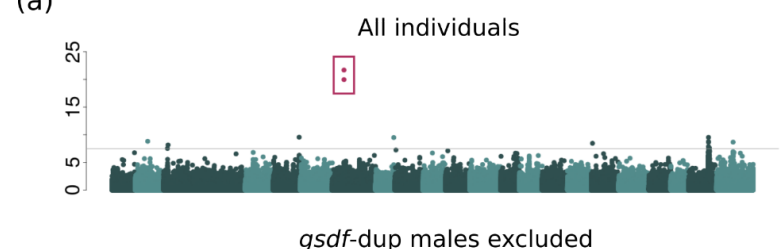

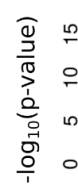

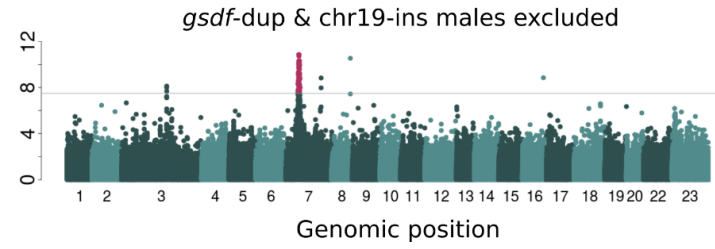

(b)

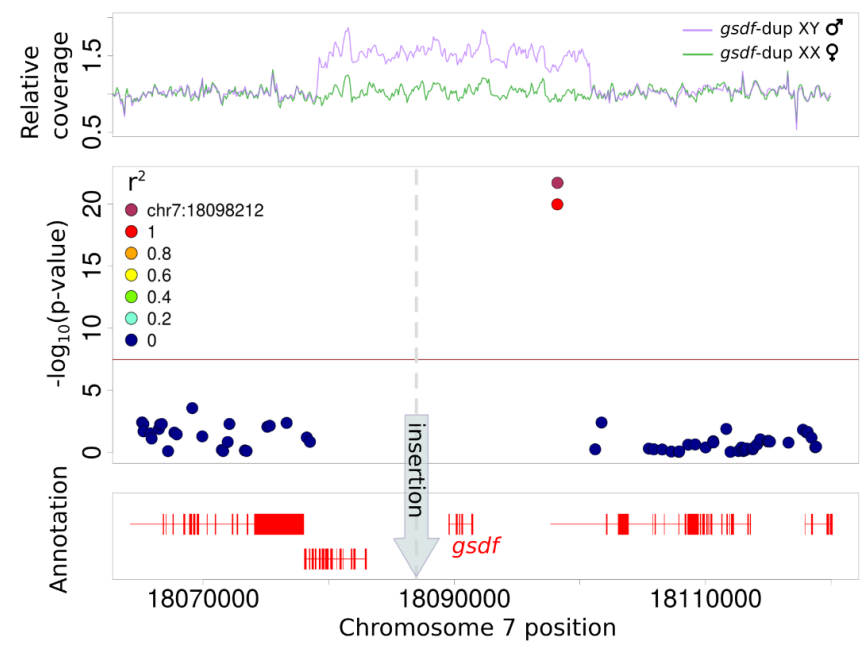

123 Figure 1: Genome-wide association study for sex. (a) P-values for the likelihood ratio test of an association between sex of Astatotliapia calliptera from Lake Masoko and their posterior mean genotypes at SNPs across the genome. The panels in order from top to bottom show results from the serial GWAS in which we looked for sex associations using all females and a subset of males not possessing the alternate allele of the single most highly-ranked SNP (or gsdf-dup specifically for iterations two and three) from any of the previous GWAS. The grey, horizontal line in each of the Manhattan plots indicates the 0.05 Bonferroni-adjusted significance threshold, correcting for the number of tested SNPS. Significant SNPs tagging sex-determining loci are shown in maroon. (b) A zoomed-in view of the region harboring the SNPs most strongly associated with sex on chromosome 7. SNPs are coloured based on their degree of linkage disequilibrium with the most strongly sex-associated SNP tagging the gsdf duplication. The top panel shows the average sequencing depth in $100 \mathrm{bp}$ bins of males heterozygous for the gsdf duplication compared to females. The sequencing depth of each individual was normalized with 6 respect to their average depth in the non-duplicated flanking regions such that an increase of $0.5 \mathrm{x}$ in males compared to females indicates the presence of an extra copy of this locus. The duplication spans the region containing the entire gsdf gene and SNPs just downstream of gsdf 9 were highly associated with sex in the GWAS run on all males and females. A $5 \mathrm{~kb}$ insertion upstream of $g s d f$ indicated by the grey arrow characterizes the chr7-ins $Y$ allele, which was in 1 high linkage with the strongly sex-associated chromosome 7 SNPs in the bottom panel of (a). 


\section{Table 1: Frequency of sex-determining genotypes in Lake Masoko Astatotilapia calliptera}

143 Multilocus genotypes for the sex determining loci are based on the number gsdf gene copies an

144 individual carries and their combination of reference (0) and insertion (1) alleles at the loci

145 characterized by the chr19-ins and chr7-ins alleles. Among the 51 females in our sample, 46

146 were classified as low PC1 and five were middle PC1, none of which carried the gsdf duplication

147 nor any of the insertion alleles.

\begin{tabular}{|c|c|c|c|c|c|c|c|}
\hline $\begin{array}{c}\text { gsdf } \\
\text { copies }\end{array}$ & $\begin{array}{c}\text { chr19-ins } \\
\text { genotype }\end{array}$ & genotype & All males & $\begin{array}{c}\text { Low PC1 } \\
\text { males }\end{array}$ & $\begin{array}{c}\text { Middle } \\
\text { PC1 } \\
\text { males }\end{array}$ & $\begin{array}{c}\text { High PC1 } \\
\text { males }\end{array}$ & Females \\
\hline 2 & $0 / 0$ & $0 / 0$ & 5 & 5 & 0 & 0 & 51 \\
\hline 3 & $0 / 0$ & $0 / 0$ & 481 & 177 & 127 & 177 & 0 \\
\hline 4 & $0 / 0$ & $0 / 0$ & 20 & 4 & 6 & 10 & 0 \\
\hline 2 & $0 / 1$ & $0 / 0$ & 59 & 38 & 21 & 0 & 0 \\
\hline 2 & $1 / 1$ & $0 / 0$ & 2 & 2 & 0 & 0 & 0 \\
\hline 2 & $0 / 0$ & $0 / 1$ & 23 & 14 & 9 & 0 & 0 \\
\hline 3 & $0 / 1$ & $0 / 0$ & 3 & 1 & 2 & 0 & 0 \\
\hline 2 & $0 / 1$ & $0 / 1$ & 1 & 1 & 0 & 0 & 0 \\
\hline 3 & missing & $0 / 0$ & 2 & 1 & 0 & 1 & 0 \\
\hline
\end{tabular}

148 The duplicated $g s d f \mathrm{Y}$ allele, which we call gsdf-dup, does not determine sex in all males: 90 of

149 the 596 males (15\%) are homozygous unduplicated, while 20 (3\%) are apparently homozygous

150 duplicated (2x relative sequence depth). To establish whether another locus might control sex in

151 the males lacking gsdf-dup, we carried out a second sex GWAS with the 51 females and 90

152 males without the duplication. This revealed a region on chromosome 19 with multiple SNPS

153 that were highly significant, the highest of which (position $21,581,905, \log _{10}$ p-value $=$

$1546.327883 \mathrm{e}-15$ ) is located $77 \mathrm{bp}$ upstream of the e2f2 gene (Figure 1a). The inferred ancestral

155 allele at this SNP was found exclusively among males across 59 heterozygotes and 3 
156 homozygotes, suggesting a second XY system (Supplemental Table 2). We inspected the

157 genomic region harboring variants in high linkage disequilibrium (LD) with the SNP to determine

158 whether it was tagging any other variants having an even stronger sex association not detected

159 by the GWAS, which was limited to biallelic SNPs. We discovered one such variant, a $700 \mathrm{bp}$

160 insertion at position 21,572,413, which is located $1.7 \mathrm{~kb}$ upstream of the id3 gene

161 (Supplementary Figure 2). This male-exclusive insertion, hereafter called chr19-ins, is found in

16262 of the 90 males without gsdf-dup, of which 60 are heterozygotes and two are homozygotes.

163 There are also three males with gsdf-dup that are heterozygous for chr19-ins. The additional

164 sequence inserted in chr19-ins occurs in 37 places across 17 chromosomes and two unplaced

165 scaffolds of the reference genome (blastn evalue = 0, > 96\% identity, 100\% coverage), and

166 matches an LTR/Unknown family transposable element (blastn evalue = 0, 97\% identity, 99\%

167 coverage) identified by repeatModeler2. At a more relaxed level of identity this transposable

168 element is found in 126 places spread across all chromosomes and eight scaffolds of the

169 reference genome (blastn evalue $=0,>92 \%$ identity, 100\% coverage).

170 Since there remain 28 males carrying neither gsdf-dup nor chr19-ins, we repeated the GWAS

171 procedure a third time, yielding another highly significant region of association on chromosome

1727 around gsdf (Figure 1a). The most significant individual SNP in this case is approximately 371

$173 \mathrm{~kb}$ upstream of $g s d f$ (position $17,718,711, \log _{10}$ p-value $=1.386670 \mathrm{e}-11$ ), with a derived allele

174 exclusively in males; 19 of the 28 males are heterozygous and one is homozygous

175 (Supplemental Table 2). This pattern is consistent with a third $Y$ allele that affects the gsdf gene

176 independently of the gsdf duplication. Further investigation in the window of elevated LD with

177 this top GWAS SNP revealed a 5 kb insertion at position 18,086,980, hereafter called chr7-ins,

178 located just $2.5 \mathrm{~kb}$ upstream of $g s d f$. This insertion is again exclusive to males including all with

179 the chr7:17718711 derived allele as well as three additional males without any previously

180 identified $Y$ allele. Two subregions of the chr7-ins sequence, one $638 \mathrm{bp}$ and the other $510 \mathrm{bp}$,

181 are respectively found at 19 and 18 places throughout 15 chromosomes and three unplaced

182 scaffolds of the $A$. calliptera reference genome (blastn evalue $=0,>90 \%$ identity, $100 \%$

183 coverage). RepeatModeler2 assigns them both to the ends of an unknown repeat family,

184 indicating that the chr7-ins insertion was also introduced by a transposable element. There

185 remain 5 males ( $0.8 \%$ of 596$)$ not carrying any of the three putative $Y$ alleles ( $g s d f$-dup,

186 chr19-ins, chr7-ins). These results showing all genotypes are summarized in Table 1. 
187 It has been reported that B chromosomes can act dominantly to determine female sex in some

188 rock-dwelling Mbuna Lake Malawi cichlids (Clark et al., 2017; 2018; 2019). We therefore

189 examined whether any of our Lake Masoko samples contained excess sequence indicative of $B$

190 chromosomes, as defined in Clark et al. (2018). None of our samples showed any such excess,

191 indicating that B chromosomes do not contribute to sex determination in this system.

192 Gsdf is expressed at higher levels in individuals carrying gsdf-affected Y alleles

193 Comparison of gene expression in the gonads of two adult male and two adult female $A$.

194 calliptera shows seven-fold higher gsdf expression in males than in females (Figure 2a),

195 consistent with observations in other fish species of higher levels of $g s d f$ in testis than ovary

196 (Zhu et al., 2018). Furthermore, male carriers of gsdf-dup and chr7-ins, the latter which could

197 plausibly be in a promoter region of $g s d f$ given its upstream proximity, express gsdf in

198 non-gonadal tissues (liver, eye, gill and anal fin) at substantially higher levels than males lacking

199 these alleles (Figure 2b \& Supplementary Figure 3). Thus, we infer that higher gsdf expression

200 resulting from more copies of the actual gene itself or changes to a regulatory element triggers

201 masculinization in Masoko A. calliptera. In contrast, the inserted chr19-ins sequence upstream

202 of $i d 3$, the nearest gene to this insertion, did not show any associated changes in expression. It

203 remains unclear how this variant results in masculinization. 
(a)

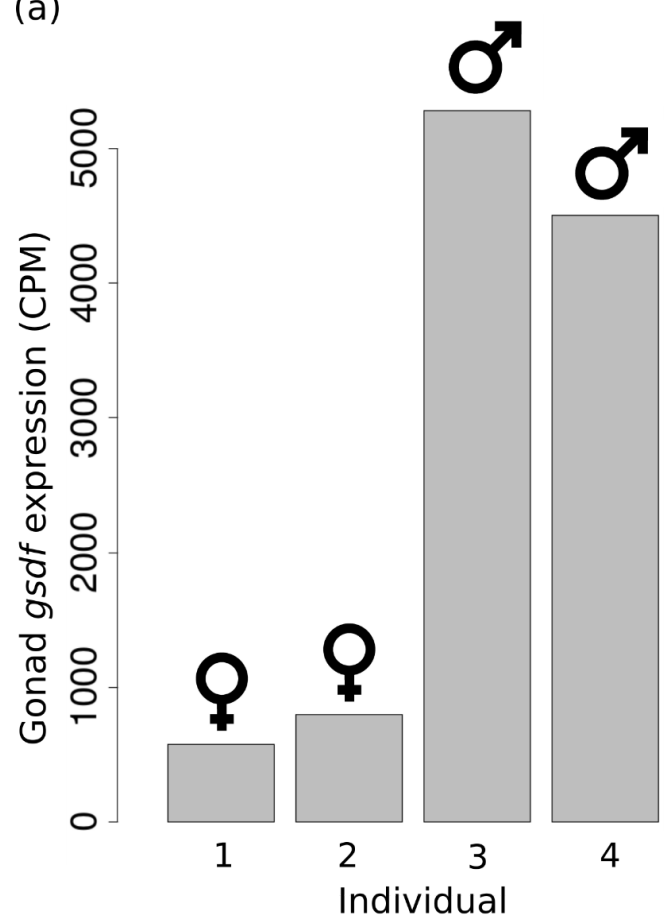

(b)

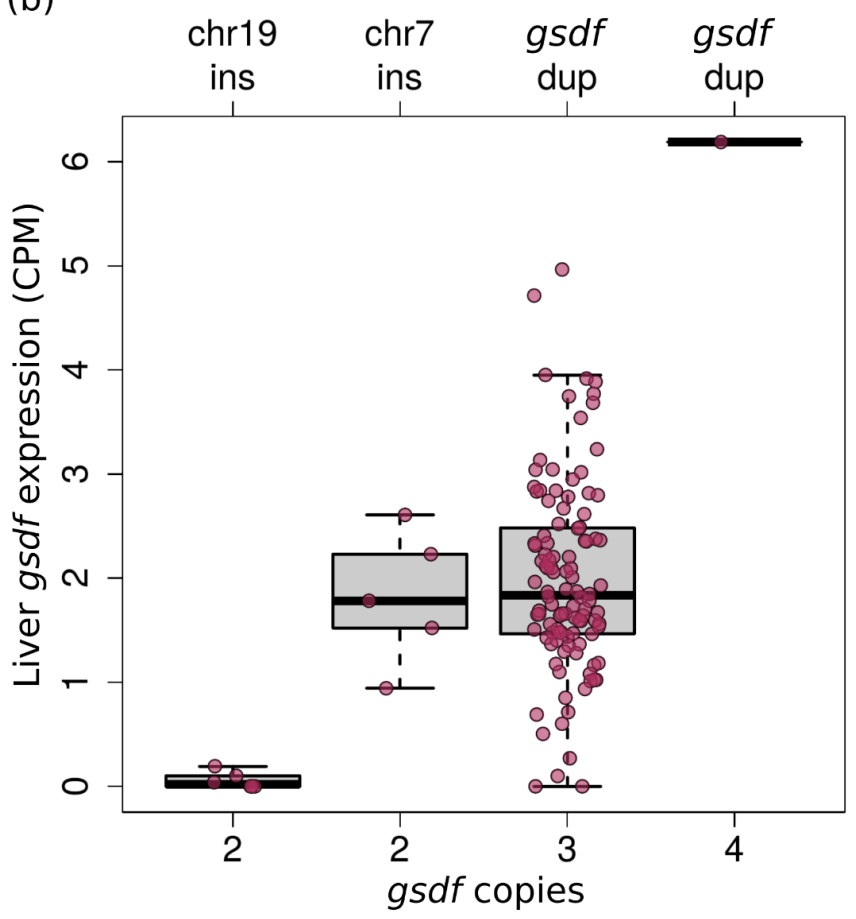

204 Figure 2: Expression of gsdf. (a) Expression levels of gsdf in the gonads of two male and two 205 female $A$. calliptera reveals approximately seven times higher gsdf expression in males. (b) 206 Comparison of gsdf expression levels in the livers of Masoko male $A$. calliptera heterozygous 207 (three copies) and homozygous (four copies) for the gsdf duplication and males lacking the 208 duplication (two copies) but who carry Y alleles generated through insertions on chromosomes 2097 and 19. The chromosome 7 insertion (chr7-ins) is directly upstream of gsdf, potentially in a 210 regulatory element of this gene. Thus, all males carrying $Y$ alleles resulting from mutations 211 thought to affect gsdf express this gene more than other males on average. Gene expression 212 was quantified as counts per million reads (CPM).

\section{Differential use of $Y$ alleles in Lake Masoko}

214 A principal component analysis (PCA) of the SNP data for the Lake Masoko samples reveals a

215 primary axis of genetic variation distinguishing the benthic from littoral ecomorph (Figure 3a), 216 and this axis is strongly correlated with catch depth (Supplementary Figure 4). There is a tight

217 cluster of samples at high principal component 1 (PC1) corresponding to the benthic ecomorph.

218 For the purposes of this paper we denote fish with PC1 > 0.4 as genetically benthic, and those 219 with PC1 < 0.4 as genetically littoral. The genetically littoral fish are more broadly distributed in 220 the PCA plot, consistent with varying degrees of benthic admixture (Supplementary Figure 5), 
221 and for some analyses below we partition them into a "low PC1" subgroup with PC1 < -0.02,

222 and a "middle PC1" group with $-0.02<$ PC1 $<0.4$.
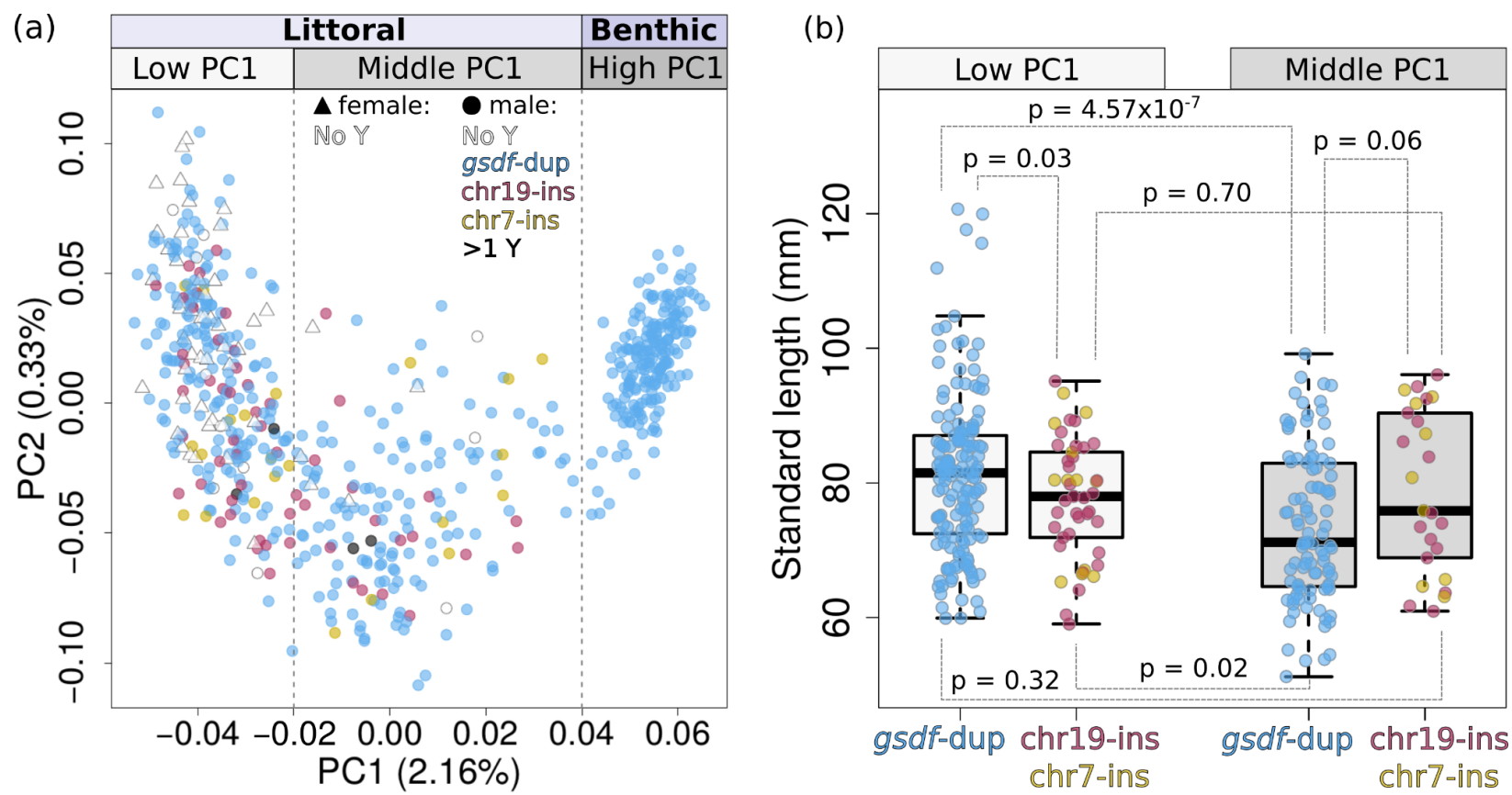

223 Figure 3: Genetic characterization of Masoko A. calliptera. (a) The first two components

224 from a principal component analysis of the genome-wide variation among $A$. calliptera from

225 Lake Masoko shows different $Y$ allele usage between fish belonging to distinct genetic clusters.

226 The points represent individuals and their colours denote which of the sex determining alleles

227 identified from the GWAS individuals carry. PC1 separates fish adhering to the benthic

228 ecomorph from littoral morph fish. The dashed grey lines show the demarcations that were used

229 to classify fish as low, middle, and high PC1, which corresponds to their level of benthic

230 ancestry across the genome. (b) Comparisons between the standard lengths of littoral males

231 heterozygous for gsdf-dup versus males heterozygous for chr19-ins or chr7-ins shows an

232 interaction between $Y$ allele type and benthic admixture levels on body size. Males carrying

233 more than one type of $\mathrm{Y}$ allele were excluded. Two-tailed t-tests were used to test for significant

234 differences between the lengths of males characterized by different genetic PC1 background

235 and $\mathrm{Y}$ allele combinations ( $\mathrm{p}$-values shown).

236 The genetically benthic fish were almost exclusively found in deep waters (> 20 metres), with

237 just three of 188 individuals at intermediate depth (5-20 metres). The genetically littoral fish

238 were found predominantly at shallow (< 5 metres) and intermediate depths, though there were 
239 some littoral fish caught in deep water, with a strong bias for these to be amongst fish with

240 higher PC1 values: in particular, amongst the 289 low PC1 subgroup individuals 138 were

241 caught shallow, 114 at intermediate depth, and 6 deep, while out of the 170 middle PC1

242 subgroup individuals 25 were caught shallow, 63 at intermediate depth, and 46 deep.

243 Interestingly, all 188 genetically benthic males carried the gsdf duplication compared to 318/408

$244(78 \%)$ of the remaining males (Figure 3a); this deviates significantly from a null hypothesis in

245 which the frequency of males using gsdf-dup is independent of PC1 $\left(x_{1}^{2}=7.35, p=0.007\right)$.

246 Correspondingly, the chr19-ins and chr7-ins alleles are only present in the genetically littoral

247 males, at respective frequencies of $8.2 \%$ and $2.9 \%$.

\section{Antagonism between $Y$ alleles and admixture}

249 Fish grow throughout life, and there is evidence that physical size is a correlate of resource

250 holding potential and reproductive success in males of African mouthbrooding cichlids

251 (Hermann et al., 2015; Nelson, 1995; Sefc, 2011) where even a $1 \mathrm{~mm}$ size difference can

252 severely impact an individual's chances of winning bouts of male-male aggression (Turner \&

253 Huntingford, 1986). In Lake Malawi haplochromine cichlids specifically, body size is a key

254 predictor of the ability to successfully hold essential breeding territory from which to court

255 females (Markert \& Arnegard 2007). Even in the absence of male-male competition, at least in

256 the case of South American convict cichlids, females prefer to mate with larger males

257 (Dechaume-Moncharmont et al., 2011), thus there is substantial evidence to suggest that male

258 cichlids may commonly benefit from being larger.

259 In Lake Masoko, the genetically littoral male fish tend to be smaller as their amount of benthic

260 ancestry increases (Supplementary Figure 6, Supplementary Table 3). This decrease in size

261 with greater benthic admixture is significantly influenced by the type of $Y$ allele that a male

262 carries (ANOVA $F=3.66, p=0.027$, comparing a linear model with interaction between genetic

263 PC1 and Y allele to a model with no interaction term). Chr19-ins males and chr7-ins males are

264 the same size in both low and middle PC1 subgroups (low PC1 two-tailed $t=-0.40, p=0.70$,

265 middle PC1 two-tailed $t=-0.24, p=0.81$ ), and together their size remains stable regardless of

266 the level of benthic ancestry (two-tailed $t=0.38, p=0.7$, Figure $3 b$ ). In contrast, gsdf-dup males

267 with middle PC1 genetic ancestry are significantly smaller than those with low PC1 ancestry

268 (two-tailed $t=5.21, p=4.57 * 10^{-7}$ ). This size difference for $g$ sdf-dup males is so pronounced that 
269 while they are significantly larger than males using the other two $Y$ alleles on the low PC1

270 background (two-tailed $t=2.24, p=0.03$ ) they tend to be smaller in an intermediate PC1

271 background. In contrast, the gsdf-dup genetically benthic (high PC1) males do not suffer from

272 the size deficit seen in gsdf-dup middle PC1 males (Supplementary Figure 7a). Males

273 homozygous for gsdf-dup are on average $81 \mathrm{~mm}$ long, which is no different than heterozygotes

274 (two-tailed $t=-0.48, p=0.64$ ), and so by this proxy are equally fit.

275 Because PC1, which reflects benthic genetic content, is correlated with fish capture depth, we

276 examined whether there could be an interaction between environment and genotype

277 contributing to these size differences. Interestingly, while the gsdf-dup males with middle PC1

278 ancestry are smaller at all catch depths, chr19-ins and chr7-ins males with middle PC1

279 backgrounds are noticeably larger at depths greater than five metres (Supplementary Figure

280 7a). This larger size of the deeper-caught chr19-ins and chr7-ins middle PC1 males is

281 counteracted by their shallow-caught counterparts tending to be the overall smallest,

282 contributing to these males appearing similar in size across genetic backgrounds when not

283 accounting for depth. Despite numbers of some categories being low, this three-way interaction

284 between the depth at which fish are caught, $\mathrm{Y}$ allele type, and level of benthic ancestry, is

285 borderline significant in its ability to predict fish length (ANOVA $F=3.02, p=0.05$ ), suggesting

286 that depth is relevant in contextualizing how different genetic combinations relate to body size,

287 and therefore fitness.

288 If the low PC1 and middle PC1 fish were sufficiently separated from each other genetically,

289 these differences in size would be expected to lead to differences in the fraction of littoral males

290 carrying the rarer insertion alleles at greater depth or PC1 values. However, a three-way

291 interaction between PC1 (restricted to low and middle PC1), catch-depth, and Y allele type is

292 not significant in modeling the frequency of males $\left(x_{2}^{2}=0.08, p=0.96\right)$, nor are interactions

293 between $Y$ allele type and depth or PC1 (Wald test $z=-0.85$ to 1.16, all p-values $>0.25$ in the

294 homogeneous association model of male frequency, which includes all pairwise interactions

295 between depth, Y allele and PC1) (Supplementary Figure 7b). Indeed, pooled across depths,

296 gsdf-dup males are 3.5x more common than males carrying either of the other two $Y$ alleles

297 among fish with low PC1 genetic backgrounds and 3.9x more common among middle PC1

298 males (difference not significant, Fisher's exact test $p=0.45$ ). 
299 Although the results of the last paragraph fail to provide direct evidence of a selective benefit for

300 the $\mathrm{Y}$ insertion alleles at deeper depths or highly admixed genetic backgrounds in terms of allele

301 frequency differences, it is noteworthy that elevated linkage disequilibrium (LD) extends for

302 hundreds to thousands of kilobases from the strongest sex-associated GWAS SNPs tagging

303 chr19-ins and chr7-ins (Supplementary Figure 2). To quantify this extent of LD we measured the

304 mean squared physical distance between the chr19-ins and chr7-ins tagging SNPs and other

305 SNPs that were within a megabase and in strong LD $\left(r^{2}>0.5\right)$ with these focal SNPs; these

306 values are in the 81st and 87th percentiles respectively compared to other randomly-sampled

307 focal SNPs across the genome with the same allele frequencies. This is consistent with

308 long-range LD generated by recent positive selection, suggesting that either the

309 sex-determining variants or another locus that they are physically linked to could be the target of

310 selection.

311 Distribution of sex-determining alleles across the Lake Malawi cichlid radiation

312 We next investigated the presence of these $Y$ alleles in other species from the Malawi radiation

313 for which we have sequenced samples. The gsdf duplication is seen in 95 additional species,

314 suggesting that it is old and may correspond to the major male-determining allele in the chr7 XY

315 system observed to act previously in multiple Lake Malawi cichlid species (Parnell \& Streelman,

316 2013; Ser et al., 2010) (Supplementary Table 5). However, its use in sex determination appears

317 to be quite dynamic; for example, it was not seen in the entire sample of $32 \mathrm{~A}$. calliptera males

318 from crater lake Itamba near to Lake Masoko (Figure 4a), and it has been lost or gained multiple

319 times within the Maylandia genus (Figure 4b). 
(a)

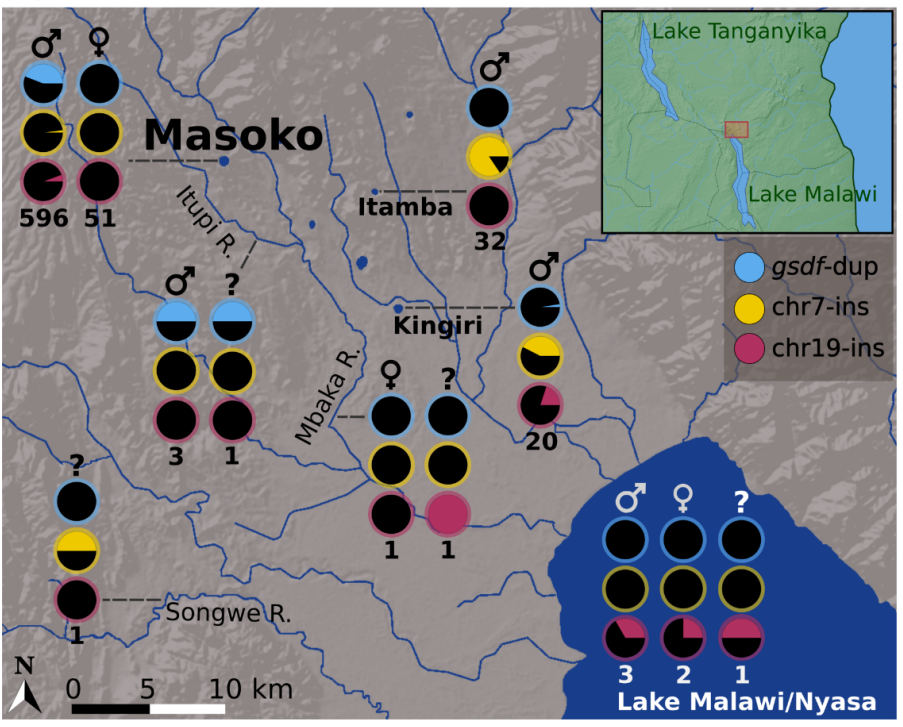

(b)

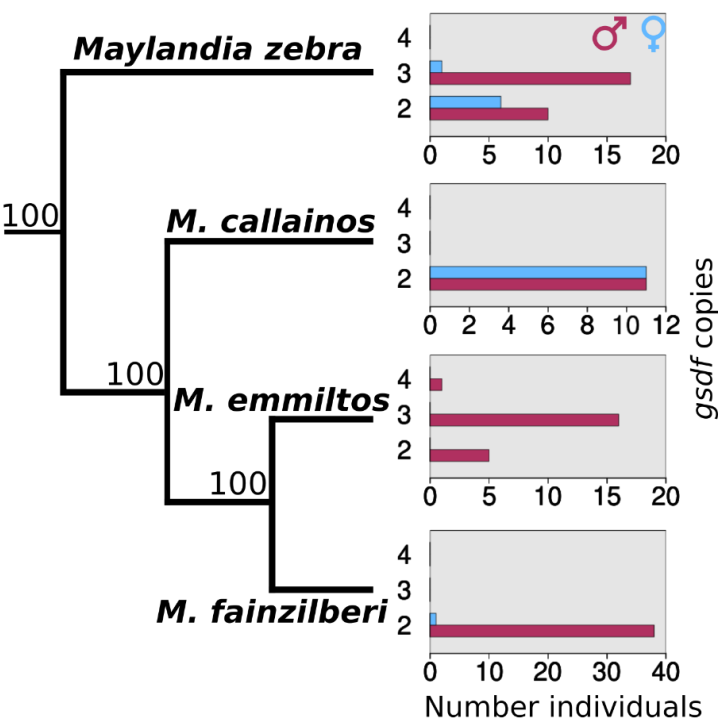

320 Figure 4: Geographic and taxonomic distribution of $Y$ alleles. (a) The frequency of the gsdf-dup, chr7-ins, and chr19-ins alleles among $A$. calliptera males, females, and individuals of unknown sex sampled from lakes and rivers throughout Tanzania and Malawi suggests varied usage of these alleles as sex determiners. The sample sizes for each sex and locality are indicated under pie charts of allele frequencies. (b) The frequency of male (blue) and female (maroon) individuals from four Maylandia species that are either heterozygous (three copy), homozygous (four copy), or lacking (two copy) the duplicated gsdf allele exemplifies the dynamic role of $g s d f$-dup in sex determination across the Malawi cichlid radiation. The presence of the gsdf duplication in relation to the neighbor-joining species tree, rooted using the distantly-related outgroup Rhamphochromis longiceps, suggests that the gsdf duplication has been lost or gained at least twice during the diversification of the Maylandia lineage. Additionally, the gsdf duplication is found in both sexes of M. zebra, although at significantly different frequencies (Fisher's exact test $p=0.035$ ), consistent with it playing a role in sex

333 determination in this population.

334 Among our specimens, the chr19-ins allele is exclusive to A. calliptera, and is geographically 335 widespread, occurring in populations from another Tanzanian crater lake, Kingiri (Figure 4a), as 336 well three other lakes, and five rivers (Supplementary Table 5) that span an area extending 337 south and north of Lake Malawi. Among the 20 non-Masoko chr19-ins carriers for which we 338 have sex information, 18 were chr19-ins heterozygote males from the Bua River and lakes 
339 Kingiri, Malombe, Chilwa, and Malawi, and two were heterozygote females from the Salima

340 population of Lake Malawi and the Ruvuma River.

341 The chr7-ins allele occurs in other lake and riverine populations of $A$. calliptera mostly from the

342 regions surrounding northern Lake Malawi except for one southern Lake Malawi population

343 (Southwest Arm). Among 20 Lake Kingiri males 55\% are heterozygous for chr7-ins and 15\% are

344 homozygous, while in 32 Lake Itamba males 31\% are heterozygous and 69\% are homozygous

345 (Figure 4a and Supplementary Table 5). The high frequency of chr7-ins homozygotes,

346 particularly in Itamba, suggests that this variant is either not sex determining or is being

347 epistatically masked by a feminizing allele in these populations. We also detected the chr7-ins

348 variant in seven species from the genus Tropheops and two Pseudotropheus species

349 (Supplementary Table 6). Both genera are endemic to Lake Malawi and belong to the Mbuna

350 clade that is phylogenetically close to A. calliptera (Malinsky et al., 2018). Small sample sizes of

351 both males and females for these species and the coincidence of both the gsdf duplication and

352 chr7-ins make it difficult to confidently discern whether chr7-ins could be involved in sex

353 determination, although there is an indication in some cases. For instance, there is one

354 Tropheops gracilior male without gsdf-dup that is heterozygous for chr7-ins while the single

355 female from this species does not carry either of these putative $Y$ alleles. Similarly, in Tropheops

356 sp. 'chilumba' and Tropheops sp. 'mauve' there are males heterozygous for chr7-ins without a

357 duplicated $g s d f$, however there are no females for comparison. Such a male is also found from

358 Tropheops sp. 'rust' but in this species, and Tropheops sp. 'white dorsal', females occur that

359 carry both gsdf-dup and chr7-ins. While sexing errors could be responsible, a potentially more

360 plausible explanation is the presence in Tropheops of a dominant female-determining variant at

361 another locus, given that females with one of or both chr7-ins and gsdf-dup are observed

362 multiple times. Of the two Pseudotropheus species positive for chr7-ins, only one,

363 Pseudotropheus fuscus, had sexed individuals; 2/2 males are heterozygous for chr7-ins and

364 have an unduplicated gsdf, while the only female lacks both gsdf-dup and chr7-ins, which is

365 consistent with chr7-ins being male-determining.

\section{Discussion}

367 Our genome-wide survey for genetic associations with sex revealed that there are three putative

$368 \mathrm{XY}$ determination systems segregating within a single natural population of Astatotilapia

369 calliptera from the crater lake Masoko. Among these, two are associated with gsdf on 
370 chromosome 7 : the duplication present in $85 \%$ of males, which is the primary mechanism, and an upstream insertion present in $4 \%$ of males. The third $Y$ allele is characterized by an insertion

372 on chromosome 19 in 11\% of males. These systems are used differentially between the

373 divergent ecomorphs in the lake, with the deep-water benthic morph only using the duplication,

374 while littoral fish use all three systems.

375 Although use of multiple sex determination systems might seem likely to create sex-ratio biases,

376 multiple $Y$ alleles can coexist without problem in a population, with each male just carrying one

377 of them, and females carrying none of them; Mendelian segregation in the offspring then gives

$37850 \%$ males with the paternal $Y$ and 50\% females. Indeed, we saw no females with any of the $Y$

379 alleles. However in our larger set of males we did detect some that carried two $Y$ alleles,

380 including males homozygous for the gsdf duplication and others with two different $Y$ alleles,

381 suggesting that there are some females carrying $\mathrm{Y}$ alleles present in the broader population. $\mathrm{A}$

382 possible explanation for this is that a dominant ZW system may also be present at low

383 frequency, in which a dominant feminizing $\mathrm{W}$ allele acts epistatically to any of the $\mathrm{Y}$ alleles, as

384 seen in some other Lake Malawi cichlid species (Parnell \& Streelman, 2013; Ser et al., 2010).

385 We did not detect such a W allele in our association scans, possibly because the number of

386 females in our data set did not give sufficient power to detect it at the frequency which would

387 explain our observations. Alternatively, there could be incomplete penetrance of the duplication

388 allele, or genetically male fish could rarely undergo environmentally-induced sex reversal, which

389 has been documented in more taxonomically distant cichlids (Baroiller et al., 1995).

390 Complete genomic sequencing of many wild individuals enabled us to identify the likely causal

391 genetic mechanisms creating new $\mathrm{Y}$ alleles and corroborate the suspicion by Peterson et al.

392 (2017) that gsdf is a sex determination locus in A. calliptera. Our findings indicate that the

393 tandem duplication of $g s d f$ and the proximal upstream insertion both boost $g s d f$ expression,

394 consistent with leading to masculinization as shown in Oryzias (Myosho et al., 2012).

395 Upregulated gsdf expression appears to be generally important for testicular development in fish

396 (Matsuda \& Sakaizumi, 2016) and gsdf has been reported as a sex determiner in multiple fish

397 species (Einfeldt et al., 2021; Jiang et al., 2016; Kaneko et al., 2015; Myosho et al., 2012).

398 Recycling of this gene for sex determination through repeated distinct mutations is evidence for

399 evolutionary conservation of the genetic pathways controlling sex even as the specific sex

400 determining alleles turn over (see Bachtrog et al. 2014 and Vicoso 2019 for discussion on this

401 topic). The second gene we identified, id3, has not previously been directly associated with sex 
402 determination, and while we believe we have identified the responsible mutation we cannot be

403 certain of the affected gene.

404 The genetic mechanisms generating the Masoko $\mathrm{Y}$ alleles parallel those involved in the origin of 405 the dmy/dmrt1bY male determining gene in Oryzias latipes, which arose from a duplication of 406 dmrt1. Two transposable elements (TEs) introduced transcription factor binding sites upstream 407 of the dmrt1b paralog, which altered its expression leading to it becoming the master 408 sex-determining gene (Herpin et al., 2010; Schartl et al., 2018). Similarly, both the chr19-ins and 409 chr7-ins $Y$ alleles were created by TE insertions directly upstream of the id3 and gsdf genes 410 respectively, offering support for the notion that TEs may play a potent role in rewiring the 411 expression of genes to function as sex determiners (Dechaud et al., 2019).

412 Usage partitioning among three different $Y$ alleles within a single, isolated population provides a 413 striking example of how dynamic sex determination is in African cichlids. This complements 414 recent work showing that across the Lake Tanganyika cichlid radiation sex systems turn over at 415 a higher rate than previously established for vertebrates (El Taher et al., 2020). Previous studies 416 showed that multiple sex determination systems can segregate within captive families involving 417 crosses between Lake Malawi species (Parnell \& Streelman, 2013; Ser et al., 2010), but did not 418 characterize their distributions within natural populations. Our results from Lake Masoko allow 419 us to explore how multiple co-occurring sex systems segregate in the wild, and their relationship 420 to subpopulation structure.

421 All of the variants that we identified for controlling sex also exist outside of Lake Masoko. The 422 presence of $g s d f$-dup across all major clades of the Lake Malawi radiation, except for

423 Diplotaxodon and Rhamphochromis, suggests that it either predated the radiation or arose early 424 in it. Despite this, the gsdf duplication has not fixed, instead showing evidence of gains and loss 425 at fine taxonomic scales within genera and even species. In contrast, chr19-ins and chr7-ins are 426 both far more taxonomically constrained, with chr19-ins exclusive to $A$. calliptera, despite being 427 widespread geographically. This suggests that these variants, although at low frequency, are 428 also old and in the case of chr7-ins could have been introduced into Tropheops and 429 Pseudotropheus through introgression. Another possibility is that chr7-ins, seen in 9/67 ( 13\%) 430 of the uniquely-classified Mbuna species (2/13 genera) in our dataset, could have arisen in a 431 common ancestor of $A$. calliptera and Mbuna and remained as a minor sex-determining player 432 in comparison to gsdf-dup, which we detected in $\sim 75 \%$ of the Mbuna species (11/14 genera). 
433 This scenario would suggest that gsdf-dup may be selectively advantageous over chr7-ins in

434 most circumstances, while there are some conditions that favour chr7-ins. A common feature of

435 all of the $Y$ alleles we identified is that outside of Masoko they do not always appear to

436 determine sex, suggesting that multifactorial sex determination is common and highly variable

437 with respect to which alleles serve as the major sex determiners, even in closely related

438 species. Having identified some of the precise variants influencing sex differentially across the

439 radiation enables future studies into the evolutionary factors supporting their turnover at a

440 variety of evolutionary scales.

441 Our results raise the question of which eco-evolutionary contexts promote the invasion and

442 eventual maintenance or loss of new sex determining variants. Theorized evolutionary

443 mechanisms contributing to sex system turnover include resolving sexually antagonistic traits

444 (van Doorn \& Kirkpatrick, 2007), escape from deleterious mutational load (Blaser et al., 2013),

445 selection on sex ratios (Eshel, 1975), genetic drift (Saunders et al., 2018), and transmission

446 distortion (Clark \& Kocher, 2019; Werren \& Beukeboom, 1998). In considering how our findings

447 align with such models it is important to recognize that we are only observing a snapshot of

448 whatever dynamics may be occurring in Masoko, rather than seeing the evolutionary trajectories

449 of $Y$ allele usage.

450 Under the classic model of sexually antagonistic selection (van Doorn \& Kirkpatrick, 2007), 451 autosomal alleles with differential fitness effects between sexes gain an advantage if they

452 become linked to a new sex determination locus, thus coupling the male-benefiting allele with

453 males and vice versa. The resulting linkage disequilibrium can be reinforced in the long term

454 through reduced recombination in the region containing the sex-determining and sexually

455 antagonistic loci. When multiple sex loci co-occur in a population as in our case, the $\mathrm{Y}$ allele

456 conferring the greatest fitness advantage to males will spread.

457 We found evidence of an antagonistic relationship in terms of body size between the different $Y$

458 alleles and genetic PC1 in littoral males. In cichlids, larger size confers higher fitness to males

459 by providing them with an advantage in defending spawning sites and procuring access to

460 reproductively active females (Hermann et al., 2015). In the shallow waters where spawning

461 littoral fish have been observed, the frequencies of males characterized by different

462 combinations of $Y$ alleles and levels of benthic ancestry correlate well with their average size:

463 gsdf-dup males with low benthic ancestry (low PC1) are largest and most common compared to 
464 males that either carry the chr19-ins or chr7-ins $Y$ alleles or have more benthic ancestry (middle

465 PC1). This suggests that in shallow water among males with low levels of benthic ancestry, 466 gsdf-dup males have a fitness advantage over males that carry the rarer $Y$ alleles. This size

467 advantage disappears however in fish with an increased benthic ancestry component, with

468 middle PC1 gsdf-dup males being smaller by nearly $8 \mathrm{~mm}$ on average. Furthermore, in waters

469 deeper than five metres, among the fish with middle PC1 ancestry, chr19 and chr7 insertion

470 males actually gain a size advantage over gsdf-dup males. These size differences are all

471 greater than the level known to be sufficient for preventing smaller males of another African

472 cichlid species from being able to effectively compete for territories (Turner \& Huntingford,

473 1986). In A. calliptera specifically, body size has been shown to significantly influence

474 male-male aggression, presumably because it signals the resource holding potential of

475 competing males (Theis et al., 2015). Therefore, we suggest that the insertion $Y$ alleles may be

476 maintained in the population by a relative advantage under these depth and genetic background

477 conditions, while there is sufficient genetic mixing between the low and middle PC1 subgroups

478 of littorals to prevent establishment of significant allele frequency differences.

479 We suggest two possible reasons, not mutually exclusive, for why the chr7-ins and chr19-ins $Y$ 480 alleles are not seen in the high PC1 benthic ecomorph. The first is that the PCA and admixture 481 plots (Figure 3a, Supplementary Figures 4, 5) are consistent with an asymmetry of gene flow 482 between the benthic and littoral ecomorphs, with the benthic ecomorph that is adapted to the 483 cold, hypoxic environment at the bottom of the lake being genetically isolated with little if any 484 gene flow from littorals into it, whereas there is gene flow from the benthics into littorals. This 485 supports the cline of benthic admixture reflected in PC1 variation amongst the littorals. Second, 486 even if there is hybridisation leading to low levels of gene flow into benthics, there are reasons 487 to suggest it is sex-biased involving littoral females and benthic males. We never caught 488 genetically benthic fish in the shallow depths where littorals breed, but we do see occasional 489 genetic littorals in deep water. Benthic males appear to exclusively use the deep water mating 490 territories that have been observed at the base of the crater wall, and we suggest that littoral 491 males may be unable to compete successfully in this forbidding environment to which they are 492 not adapted whereas littoral females may accept mating. In this scenario low frequency $Y$ alleles 493 from the littorals would not invade the benthics at an appreciable rate, and any that were 494 present in the founders or entered through rare hybridization events could have been easily lost 495 by drift. 
496 In conclusion, our discovery that at least three different alleles control sex and segregate

497 differentially within an isolated population of $A$. calliptera provides evidence that genetic sex

498 determination in nature can be extremely fluid even at very small demographic scales. All of the

499 alleles we identified involved structural genetic variants, with two of the three generated by

500 transposable element insertions, highlighting a potentially important role for TEs in the rapidly

501 evolving sex systems of African cichlids, similar to their role in adaptive variation in opsin

502 regulation (Carleton et al. 2020). Our results also indicate that genetic background differences

503 likely created by admixture can bring about antagonistic relationships among males carrying

504 different $Y$ alleles, providing an evolutionary context that may favour multifactorial sex systems.

505 This has interesting implications for the incipient speciation between littoral and benthic Masoko

506 ecomorphs in that alternative $\mathrm{Y}$ alleles circumvent negative genetic interactions brought about

507 by admixture, allowing for sustained back-crossing that reduces the level of divergence. It is

508 possible that this contributes to the low genome-wide $\mathrm{F}_{\mathrm{ST}}(4 \%)$ between the ecomorphs, which

509 also lack fixed genetic differences, although there are tens of islands of high $\mathrm{F}_{\mathrm{ST}}$ divergence

510 potentially associated with loci under differential selection (Malinsky et al., 2015). Admixture and

511 relatively low divergence are hallmarks of the Malawi cichlid radiation, so it seems plausible that

512 similar processes could exist or have existed elsewhere. The fact that we and other studies

513 have found polygenic sex determination systems that differ markedly between closely related

514 species and populations across the radiation supports this possibility.

\section{Methods}

\section{Samples and sequencing}

517 Fish were primarily collected by professional aquarium fish catching teams. Fish at a target

518 depth range (determined by diver depth gauges) were chased into block nets by SCUBA divers

519 and transferred to a holding drum, then brought to the surface, where they were euthanized with

520 clove oil. The right pectoral fin of sampled individuals was then removed and stored in ethanol,

521 and the remainder of the specimen pinned, photographed, labelled and preserved in ethanol for

522 later morphological analysis. Standard lengths were measured using calipers. Females were

523 distinguished from juvenile males among the smaller fish by visual inspection of the gonads

524 after opening the abdominal cavity. Adult males were identified from secondary sexual traits of

525 larger size, brighter colour and possession of elongate filaments on the pelvic, dorsal and anal 
526 fins (confirmed to be reliable by visual inspection of the gonads in a number of specimens from

527 earlier collections).

528

546 using FastQC 0.11 .8 (https://www.bioinformatics.babraham.ac.uk/projects/fastqc/). We also

547 extracted RNA from the anal fins, eyes, gills and livers of $151 \mathrm{~A}$. calliptera collected from Lake

548 Masoko in 2015, 2016 and 2018 (Supplementary Table 1), which was stored in RNALater, using

549 Direct-zol TM RNA MiniPrep Plus kits (Zymo, R2072) with an additional Chloroform step before

550 loading the sample onto filtration columns. RNA samples were quantified with the Qubit ${ }^{\mathrm{TM}}$ RNA

551 HS Assay Kit and quality assessed on the Agilent 4200 TapeStation. Libraries were prepared

552 using Illumina mRNA sequencing kits with polyA enrichment and sequenced using 100 or 150

553 bp paired-end reads on three lanes of the Illumina HiSeq4000 and five S4 lanes of the Illumina

554 NovaSeq. Adapter sequences and bases with Phred quality below 20 were removed from the

555 ends of all resulting RNAseq reads using Trim Galore 0.6.4 and read quality was checked using

556 FastQC 0.11.9. 
558 Sequencing reads for all $A$. calliptera samples were mapped to a high-quality $A$. calliptera

559 reference genome (fAstCal1.2, accession GCA_900246225.3) (Rhie et al., 2021) using

560 bwa-mem 0.7.17 (Li, 2013). We used GATK 3.8 (McKenna et al., 2010) to identify

561 individual-level variation with the HaplotypeCaller program followed by joint genotype calling

562 among all samples using GenotypeGVCFs (Poplin et al., 2017; Van der Auwera \& O'Connor,

563 2020). Sites exhibiting any of the following indications of quality issues in the medium-coverage

564 ( 15x) "cichl" subset of 336 individuals were masked from all analyses: total sequencing depth

565 across individuals more extreme than the genome-wide median total site depth (DP) $+/-25 \%$,

566 fewer than $90 \%$ of individuals covered by at least eight reads, more than $10 \%$ of individuals with

567 missing genotypes, root mean square mapping quality less than 40 , an alternate allele assertion

568 quality score below 30 , a variant quality by depth score below three, excess heterozygosity

569 (exact test $p$-value $<1 e-4$ ), biases between reference and alternate alleles in terms of strand

570 (exact test $p$-value $<1 e-6$ ), base quality (z-score $>6$ ), mapping quality (z-score $>6$ ), and read

571 position (z-score $>6$ ). Sites spanning indels or having more than two alleles were also masked

572 from analyses. Quality control for sites was carried out using the program vcfCleaner

573 (https://github.com/tplinderoth/ngsQC/tree/master/vcfCleaner).

574 Population genetic characterization

575 We used principal component analysis (PCA) based on genotype posterior probabilities at the

576 quality-controlled SNPs to characterize the distribution of $A$. calliptera genetic variation

577 throughout Lake Masoko. Specifically, we used ANGSD 0.929 (Korneliussen et al., 2014) to

578 estimate minor allele frequencies from genotype likelihoods (-GL 1 model) calculated using

579 reads with minimum base and map Phred qualities of at least 20 . These minor allele frequency

580 (MAF) estimates and genotype likelihoods were used to obtain genotype posterior probabilities

581 for all individuals under a Hardy-Weinberg genotype prior. We used ngsCovar 1.0.2 (Fumagalli

582 et al., 2014) to estimate the genetic covariance matrix among individuals based on their

583 genotype posteriors at SNPs with MAF greater than 5\%, which we decomposed in R 3.6.3 (R

584 Core Team, 2020) with the eigen() function. In addition, we used the program ADMIXTURE

585 1.3.0 (Alexander et al., 2009) to infer the proportions of distinct genetic ancestry for individuals

586 assuming two ancestral populations (K parameter). 
588 We relaxed some quality filters to accept additional biallelic SNPs for statistical association 589 testing by requiring that they have a minimum total depth across individuals of 2000x (lowered 590 from 3500x), at least 90\% of individuals covered by a minimum of four reads, and an exact test 591 p-value for excess heterozygosity above 1e-20. All other quality criteria were kept the same. We 592 queried all such SNPs across the genome with MAF of at least $5 \%$ for association with sex 593 under the linear mixed model framework implemented in GEMMA 0.98.1 (Zhou \& Stephens, 594 2012). Sex was treated as a binary response which we regressed against posterior mean 595 genotypes calculated from the GATK genotype likelihoods using vcf2bimbam 596 (https://github.com/tplinderoth/ngsQC/tree/master/vcfCleaner) under a Hardy-Weinberg 597 genotype prior. We accounted for confounding effects of ancestry among individuals through 598 incorporating a centered pairwise kinship matrix calculated using GEMMA as a random effect in 599 the LMM. We identified significantly associated loci using the likelihood-ratio test p-values from 600 GEMMA run in the LMM mode at a 5\% significance level after a Bonferroni correction for the 601 number of tested SNPs. In order to identify as many sex-associated loci as possible, we 602 iteratively tested conditional subsets of individuals who did not carry alleles significantly 603 associated with sex from previous iterations, that is, subsets of individuals whose sex was not 604 accounted for by other candidates.

605 Characterizing sex-determining variants throughout Lake Masoko and the Malawi radiation

606 We only used SNPs with GEMMA and so following the sex GWAS we checked for the presence 607 of structural variants (SVs) that might have a stronger association with sex in $10 \mathrm{~kb}$ windows 608 extending from the significantly associated SNPs. We extracted read mapping information 609 directly from the BAM files to look for mapping signatures that would be consistent with 610 structural variation, considering both read pair and depth information, using IGV 2.8.0 (Robinson 611 et al., 2011). We initially screened at least five males and five females for structural variation in

612 IGV and then used a custom perl script to call SVs if at least $5 \%$ of read pairs among all

613 individuals within 480 bp of any putative SV positions had mates which mapped to a different

614 chromosome. We assembled the anomalously mapped read pairs across all individuals for each

615 SV that we called using MEGAHIT 1.2.9 (Li et al., 2016) and performed a blastn (Altschul et al., 616 1990; Camacho et al., 2009) search of the resulting contigs against fAstCal1.2. This approach 
617 led to the discovery of the putative sex-determining insertions on chromosomes 7 and 19, which

618 blasted with at least $90 \%$ identity across their full length to multiple places across the genome.

619 We used repeatModeler2 2.0.2 (Flynn et al., 2020) with default options but including the

620 -LTRStruct option to identify transposable element sequences in the fAstCal1.2 genome. Then

621 we compared the SV contigs to these transposable element sequences to further characterize

622 the insertions. The chr19-ins allele matched a 700 bp transposable element (blastn evalue $=0$,

$62397 \%$ identity, 99\% coverage) identified by repeatModeler2 as belonging to an LTR/Unknown

624 family. The two partial contigs of the chromosome 7 insertion matched with $94 \%$ identity

625 (631/673 bp with 35/673 bp (5\%) gaps) and 97\% (496/509 bp with 11/509 bp (2\%) gaps) to

626 either end of a 3,947 bp unknown transposable element.

627 In order to characterize the presence or absence of the chromosome 7 and 19 insertions, we

628 mapped sequencing reads from all Masoko $A$. calliptera to the assembled insertion sequences

629 including $1 \mathrm{~kb}$ of upstream and downstream flanking sequence using BWA. We considered any

630 reads mapping within the flanking regions and which spanned the insertion as reference allele

631 reads (with respect to fAstCal1.2) and any reads which mapped within the insertion by a

632 minimum of three bp as alternate allele reads. An individual's genotype was called

633 heterozygous (0/1) if they possessed reads from both alleles that were each at a minimum

634 frequency of $10 \%$, otherwise, with more than $90 \%$ of either the reference or insertion reads,

635 individuals were called as homozygous for the reference allele (0/0) or homozygous for the

636 insertion allele (1/1), respectively. We also genotyped fish based on the copy number of the

637 duplicated gsdf-containing locus which spans positions $18,079,155$ to $18,100,834$ of

638 chromosome 7 in the fAstCal1.2 reference. For each individual, we translated their average

639 sequencing depth across this region relative to their average sequencing depth from 38,320 bp

640 flanking sequence $(19,154$ bp upstream and 19,166 bp downstream of the duplication

641 breakpoints) into copy number in increments of $0.5 x$ : Relative coverage of 1.25 or lower was

642 recorded as a non-duplicated gsdf region, $(1.25,1.75]$ as three $g s d f$ copies, $(1.75,2.25]$ as four

643 copies, and so on. Individuals with three and four copies of the gsdf locus were called

644 heterozygous and homozygous for the duplication respectively. Though it is possible for a

645 four-copy individual to have one chromosome with three gsdf copies this would necessitate

646 another duplication and so is less parsimonious than the assumption that they are homozygous

647 for a chromosome with two copies. 
648 We also developed a PCR assay for the gsdf duplication (Supplementary Table 7), which we

649 used to confirm its presence in a subset of $A$. calliptera and Maylandia zebra. Genomic DNA

650 was extracted from fin clips using PureLink Genomic DNA Mini Kits (ThermoFisher Scientific,

$651 \mathrm{~K} 182001)$ following the manufacturer's protocols and eluted in 30-60 $\mu \mathrm{L}$ elution buffer. We

652 carried out PCRs in $20 \mu \mathrm{L}$ reaction volumes consisting of $1 \mathrm{X}$ Platinum ${ }^{\mathrm{TM}}$ II PCR Buffer, $0.2 \mathrm{mM}$

653 of each dNTP (ThermoFisher Scientific, R0192), $0.2 \mu \mathrm{M}$ of each primer (Merck Life Science,

654 desalted), less than $500 \mathrm{ng}$ template DNA (1 $\mu \mathrm{L}$ genomic DNA at $\sim 1-5 \mathrm{ng} / \mu \mathrm{L}$ ), $0.04 \mathrm{U} / \mu \mathrm{L}$

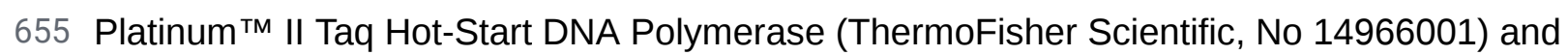

656 nuclease-free water. We amplified the DNA using the following thermal profile: $94^{\circ} \mathrm{C}$ for two

657 minutes followed by $30-35$ cycles of $94^{\circ} \mathrm{C}$ for 15 seconds, $60^{\circ} \mathrm{C}$ for 15 seconds, $68^{\circ} \mathrm{C}$ for 15

658 seconds, and a final $68^{\circ} \mathrm{C}$ extension for five minutes. The PCR products were separated using

659 electrophoresis run at 100 volts for 30 minutes on a $2 \%$ agarose gel.

660 We genotyped 1,552 additional individuals from all seven of the Lake Malawi radiation clades

661 (A. calliptera, Mbuna, Benthic, Deep, Utaka, Diplotaxodon, and Rhamphochromis; see Malinsky

662 et al. 2018) for the gsdf duplication as well as the chromosome 7 and 19 insertions in the same

663 way as for Masoko A. calliptera described above. This set of Malawi radiation individuals

664 represents 255 species (some are not formally established but recognized as distinct taxa) from

66547 genera, including A. calliptera from locations other than Lake Masoko. In order to

666 characterize how the gsdf duplication is acquired and lost as lineages diversify we mapped its

667 presence at different copy number in males and females to the species tree for four Mbuna

668 species from the Maylandia genus: M. zebra, M. callainos, M. emmiltos, and M. fainzilberi. We

669 generated the species tree using 12,133,030 genome-wide segregating sites among the four

670 Maylandia species identified using GATK 3.8 in the same manner as for Masoko A. calliptera.

671 These SNPs passed quality controls addressing abnormally low and high sequencing coverage

672 and low mapping quality for the ingroup samples as well as for samples from the

673 distantly-related species Rhamphochromis longiceps, which served as an outgroup. We used

674 ngsDist 1.0.8 (Vieira et al., 2016) to calculate a pairwise genetic distance matrix based on

675 genotype likelihoods for all of the ingroup and outgroup samples, as well as to bootstrap sites in

676 order to generate 100 additional bootstrap distance matrices. For this Maylandia species tree,

677 we used fastME 2.1.6.1 (Lefort et al., 2015) to infer neighbor-joining trees from the genetic

678 distance matrices using the BIONJ algorithm with SPR tree topology improvement. RAxML-NG

679 1.0.1 (Kozlov et al., 2019) was used to determine the bootstrap support for the genome-wide

680 tree. 
682 In addition to autosomal sex loci, B chromosomes, which are supernumerary chromosomes not

683 required for organismal function and variably present across taxa and individuals, have been

684 implicated as sex modifiers in Lake Malawi cichlids (Clark et al., 2017). Accordingly, we assayed

685 for the presence of $B$ chromosomes among Masoko A. calliptera to discern whether they may

686 influence sex. B chromosome material initially derives from autosomes, so their presence can

687 be detected through inflated read coverage in homologous regions of the reference genome

688 where B reads mismap. Accordingly, we assayed for B chromosomes based on inflated

689 coverage at regions containing sequence known to exist on B chromosomes from Lake Malawi

690 cichlids (Clark et al., 2018). Regions identified as core B block sequence according to Clark et

691 al. (2018) were translated into fAstCal1.2 coordinates and the mean coverage across each of

692 these segments for each Masoko A. calliptera individual was calculated directly from the BAM

693 files. We used a minimum coverage ratio for the core $B$ region compared to the genome-wide

694 average of $2 x$ to call B positive individuals. None of the Lake Masoko A. calliptera passed this

695 threshold although this process did identify individuals carrying B chromosomes from other

696 species.

697 Expression of sex-associated genes

698 We mapped the quality-controlled liver, eye, gill, and anal fin RNAseq reads to the fAstCal1.2

699 genome with STAR 2.7.3a (Dobin \& Gingeras, 2015) and counted reads derived from

700 sex-associated genes with featureCounts 2.0.1 (Liao et al., 2014). These read counts were

701 normalized to counts per million (CPM) reads using edgeR 3.30.3 (Robinson et al., 2010). We

702 mapped the quality-controlled gonad reads to the fAstCal1.2 reference using bwa-mem and

703 counted reads derived from gsdf exons using SAMtools 1.9 (Li et al., 2009) and ngsAssociation

704 0.2.4 (https://github.com/tplinderoth/ngsAssociation) summarize, which were also normalized to

705 CPM.

706 Relationship between Y alleles and body size

707 Genetic PC1 was used as a proxy for the degree of admixture since this component clearly

708 separates fish based on their degree of benthic ancestry. Based on distinct clustering in the 
709 genome-wide PCA plot, fish with PC1 > 0.04 were classified as genetically benthic and those

710 with PC1 < 0.04 as genetically littoral. We further classified fish with the lowest amounts of

711 benthic ancestry as "low PC1" (PC1 <-0.02), those with more equal amounts of littoral and

712 benthic ancestry as "middle PC1" (PC1 range -0.02 to 0.04), and the clear benthic cluster as

713 "high PC1" (PC1 > 0.04). The three $Y$ alleles segregate in the littoral group only, which is

714 composed of low and middle PC1 fish, yielding six possible $\mathrm{Y}$ and PC1 combinations when

715 excluding the $0.7 \%$ of males that carry more than one type of Y. For all analyses related to fish

716 size we considered only males that were heterozygous for their $Y$ allele (except when we

717 compared the length of gsdf-dup homozygotes to gsdf-dup heterozygotes). We tested the

718 hypothesis that littoral Lake Masoko A. calliptera males with different ancestry backgrounds and

$719 \mathrm{Y}$ allele combinations differ in standard length using pairwise two-tailed t-tests in R.

720 We investigated whether the size of littoral males is influenced by interactions between $Y$ allele

721 and ancestry regime by fitting linear models of standard length as a function of $Y$ allele and PC1

722 class in R using $\mathrm{glm}()$. We tested whether the interaction provides a significantly better fit with

723 the anova() F-test by comparing the residual sums of squares between a model with only main

724 effects to a model with main effects and an interaction between $Y$ allele type and PC1 class. We

725 also introduced a depth class variable into our models to investigate whether the depth at which

726 fish were caught plays a role in explaining their length. Depths less than five metres were

727 considered "shallow", depths ranging from 5-20 metres were "intermediate", and depths more

728 than 20 metres were "deep". As before, we compared the fit of a saturated model including the

729 three-way interaction between $\mathrm{Y}$ allele, PC1 class, and depth band to the same model but

730 without the three-way interaction using analysis of variance to determine if the joint interaction

731 between all variables provides a significant amount of additional power for predicting fish length.

732 Since the size of male fish is likely to influence fitness, we used log-linear models to look at

733 whether the same factors affecting length could predict the frequency of males. Specifically, we

734 fit models using $\mathrm{glm}()$ in R with family='poisson' for the frequency of males based on $\mathrm{Y}$ allele,

735 PC1 class, and depth band. We assessed whether the frequency of males belonging to

736 categories based on these three variables are independent of one another, and if not, what

737 interactions were involved by performing an analysis of variance on nested pairs of models. We

738 tested whether the differences in the residual deviance between the models being compared

739 were significant using $x^{2}$ tests. This enabled us to find the simplest model that predicts male

740 frequencies statistically as well as the saturated model that includes all main effects and their 
741 possible interactions. The significance of terms within the context of a particular model for which

742 they were fit was determined using a Wald test of the null hypothesis that a term's effect is equal

743 to zero.

744 Assessment of linkage disequilibrium around sex loci

745 We calculated LD in terms of $r^{2}$ between each of the most highly sex-associated GWAS SNPS

746 and their surrounding SNPs using PLINK 1.9 (Purcell, 2014; Purcell et al., 2007). We observed

747 high LD, $r^{2}>0.5$, between the strongest GWAS SNPs tagging chr19-ins and chr7-ins and

748 far-ranging surrounding SNPs, which we visualized using plot_zoom

749 (https://github.com/hmunby/plot_zoom). In order to determine how unusual these long stretches

750 of high LD were, we compared the variance in the pairwise physical distance between the top

751 GWAS SNPs and all SNPs within one megabase and $r^{2}>0.5$ to an expected distribution. The

752 background distributions were generated by randomly sampling 5,000 focal SNPs from across

753 the genome having the same alternate allele frequencies as each of the top GWAS SNPs. For

754 each sampled SNP, we calculated the variance among pairwise distances with other SNPs in

755 the same way as we had done for the GWAS SNPS.

\section{Acknowledgments}

757 We are grateful to African collaborators who assisted in sample collection, particularly the staff

758 of the Tanzanian Fisheries Research Institute, as well as Alan Hudson. We thank the

759 sequencing core staff at the Wellcome Sanger Institute. This work was supported by the

760 Wellcome Trust (WT207492 and WT206194). Additional support was to MJG \& GFT

761 Leverhulme Trust - Royal Society Africa Awards (AA100023 and AA130107); to MJG

762 Leverhulme Trust award (RF-2014-686); to GFT Leverhulme Trust award (RPG-2014-214); to

763 EAM Wellcome Trust Senior Investigator award (104640/Z/14/Z and 219475/Z/19/Z) and CRUK

764 award (C13474/A27826). GV thanks Wolfson College, University of Cambridge and the

765 Genetics Society, London for financial support.

\section{Competing interests}

767 The authors declare that they have no competing interests. 


\section{References}

Albertson, R. (2002). Genetic basis of adaptive radiation in East African cichlids [Doctoral

Alexander, D. H., Novembre, J., \& Lange, K. (2009). Fast model-based estimation of ancestry in unrelated individuals. Genome Research, 19(9), 1655-1664. https://doi.org/10.1101/gr.094052.109

Altschul, S. F., Gish, W., Miller, W., Myers, E. W., \& Lipman, D. J. (1990). Basic local alignment search tool. Journal of Molecular Biology, 215(3), 403-410. https://doi.org/10.1016/S0022-2836(05)80360-2

Bachtrog, D., Mank, J. E., Peichel, C. L., Kirkpatrick, M., Otto, S. P., Ashman, T.-L., Hahn, M. W., Kitano, J., Mayrose, I., Ming, R., Perrin, N., Ross, L., Valenzuela, N., Vamosi, J. C., \& Tree of Sex Consortium. (2014). Sex determination: Why so many ways of doing it? PLoS Biology, 12(7), e1001899. https://doi.org/10.1371/journal.pbio.1001899

Baroiller, J. F., Chourrout, D., Fostier, A., \& Jalabert, B. (1995). Temperature and sex chromosomes govern sex ratios of the mouthbrooding Cichlid fish Oreochromis niloticus. Journal of Experimental Zoology, 273(3), 216-223. https://doi.org/10.1002/jez.1402730306

Bezault, E., Clota, F., Derivaz, M., Chevassus, B., \& Baroiller, J.-F. (2007). Sex determination and temperature-induced sex differentiation in three natural populations of Nile tilapia (Oreochromis niloticus) adapted to extreme temperature conditions. Aquaculture, 272, S3-S16. https://doi.org/10.1016/j.aquaculture.2007.07.227

Blaser, O., Grossen, C., Neuenschwander, S., \& Perrin, N. (2013). Sex-chromosome turnovers induced by deleterious mutation load. Evolution, 67(3), 635-645. https://doi.org/10.1111/j.1558-5646.2012.01810.x

Brawand, D., Wagner, C. E., Li, Y. I., Malinsky, M., Keller, I., Fan, S., Simakov, O., Ng, A. Y., Lim, Z. W., Bezault, E., Turner-Maier, J., Johnson, J., Alcazar, R., Noh, H. J., Russell, P., Aken, B., Alföldi, J., Amemiya, C., Azzouzi, N., ... Di Palma, F. (2014). The genomic substrate for adaptive radiation in African cichlid fish. Nature, 513(7518), 375-381. https://doi.org/10.1038/nature13726

Camacho, C., Coulouris, G., Avagyan, V., Ma, N., Papadopoulos, J., Bealer, K., \& Madden, T. L. (2009). BLAST+: Architecture and applications. BMC Bioinformatics, 10(1), 421. https://doi.org/10.1186/1471-2105-10-421

Carleton, K. L., Conte, M. A., Malinsky, M., Nandamuri, S. P., Sandkam, B. A., Meier, J. I., 
Mwaiko, S., Seehausen, O., \& Kocher, T. D. (2020). Movement of transposable elements contributes to cichlid diversity. Molecular Ecology, 29(24), 4956-4969. https://doi.org/10.1111/mec.15685

Clark, F. E., Conte, M. A., Ferreira-Bravo, I. A., Poletto, A. B., Martins, C., \& Kocher, T. D.

Dechaume-Moncharmont, F.-X., Cornuau, J. H., Keddar, I., Ihle, M., Motreuil, S., \& Cézilly, F. (2011). Rapid assessment of female preference for male size predicts subsequent choice of spawning partner in a socially monogamous cichlid fish. Comptes Rendus Biologies, 334(12), 906-910. https://doi.org/10.1016/j.crvi.2011.08.004 (2017). Dynamic Sequence Evolution of a Sex-Associated B Chromosome in Lake Malawi Cichlid Fish. Journal of Heredity, 108(1), 53-62.

Clark, F. E., Conte, M. A., \& Kocher, T. D. (2018). Genomic Characterization of a B

Clark, F. E., \& Kocher, T. D. (2019). Changing sex for selfish gain: B chromosomes of Lake Chromosome in Lake Malawi Cichlid Fishes. Genes, 9(12).

$$
\text { https://doi.org/10.3390/genes9120610 }
$$
Malawi cichlid fish. Scientific Reports, 9(1), 20213. https://doi.org/10.1038/s41598-019-55774-8

Conte, M. A., \& Kocher, T. D. (2015). An improved genome reference for the African cichlid, Metriaclima zebra. BMC Genomics, 16(1), 724. https://doi.org/10.1186/s12864-015-1930-5

Dechaud, C., Volff, J.-N., Schartl, M., \& Naville, M. (2019). Sex and the TEs: Transposable elements in sexual development and function in animals. Mobile DNA, 10(1), 42. https://doi.org/10.1186/s13100-019-0185-0

833 El Taher, A. E., Ronco, F., Matschiner, M., Salzburger, W., \& Böhne, A. (2020). Dynamics of sex chromosome evolution in a rapid radiation of cichlid fishes [Preprint]. bioRxiv. 
Flynn, J. M., Hubley, R., Goubert, C., Rosen, J., Clark, A. G., Feschotte, C., \& Smit, A. F. (2020). RepeatModeler2 for automated genomic discovery of transposable element families. Proceedings of the National Academy of Sciences of the United States of America, 117(17), 9451-9457. https://doi.org/10.1073/pnas.1921046117

854 Holzberg, S. (1978). A field and laboratory study of the behaviour and ecology of Pseudotropheus zebra (Boulenger), an endemic cichlid of Lake Malawi (Pisces; Cichlidae). Journal of Zoological Systematics and Evolutionary Research, 16(3), 171-187. https://doi.org/10.1111/j.1439-0469.1978.tb00929.x

858 Jiang, D. N., Yang, H. H., Li, M. H., Shi, H. J., Zhang, X. B., \& Wang, D. S. (2016). gsdf is a downstream gene of dmrt1 that functions in the male sex determination pathway of the Nile tilapia. Molecular Reproduction and Development, 83(6), 497-508.

https.//doi.org/10.1002/mrd.22642

862 Kaneko, H., Ijiri, S., Kobayashi, T., Izumi, H., Kuramochi, Y., Wang, D.-S., Mizuno, S., \& 863 Nagahama, Y. (2015). Gonadal soma-derived factor (gsdf), a TGF-beta superfamily

866 Kocher, T. D. (2004). Adaptive evolution and explosive speciation: The cichlid fish model. Nature 

https://doi.org/10.1186/s12859-014-0356-4

Kozlov, A. M., Darriba, D., Flouri, T., Morel, B., \& Stamatakis, A. (2019). RAxML-NG: A fast, scalable and user-friendly tool for maximum likelihood phylogenetic inference. Bioinformatics, 35(21), 4453-4455. https://doi.org/10.1093/bioinformatics/btz305

Lande, R., Seehausen, O., \& Alphen, J. J. M. van. (2001). Mechanisms of rapid sympatric speciation by sex reversal and sexual selection in cichlid fish. Genetica, 112/113, 435-443. https://doi.org/10.1023/A:1013379521338

Lefort, V., Desper, R., \& Gascuel, O. (2015). FastME 2.0: A Comprehensive, Accurate, and Fast Distance-Based Phylogeny Inference Program. Molecular Biology and Evolution, 32(10), 2798-2800. https://doi.org/10.1093/molbev/msv150

Li, D., Luo, R., Liu, C.-M., Leung, C.-M., Ting, H.-F., Sadakane, K., Yamashita, H., \& Lam, T.-W. (2016). MEGAHIT v1.0: A fast and scalable metagenome assembler driven by advanced methodologies and community practices. Methods, 102, 3-11. https://doi.org/10.1016/j.ymeth.2016.02.020

Li, H., Handsaker, B., Wysoker, A., Fennell, T., Ruan, J., Homer, N., Marth, G., Abecasis, G., Durbin, R., \& 1000 Genome Project Data Processing Subgroup. (2009). The Sequence Alignment/Map format and SAMtools. Bioinformatics, 25(16), 2078-2079. https://doi.org/10.1093/bioinformatics/btp352

Li, Heng. (2013). Aligning sequence reads, clone sequences and assembly contigs with BWA-MEM. ArXiv:1303.3997 [q-Bio]. http://arxiv.org/abs/1303.3997

Liao, Y., Smyth, G. K., \& Shi, W. (2014). featureCounts: An efficient general purpose program for assigning sequence reads to genomic features. Bioinformatics, 30(7), 923-930. https://doi.org/10.1093/bioinformatics/btt656

Malinsky, M., Challis, R. J., Tyers, A. M., Schiffels, S., Terai, Y., Ngatunga, B. P., Miska, E. A., Durbin, R., Genner, M. J., \& Turner, G. F. (2015). Genomic islands of speciation separate cichlid ecomorphs in an East African crater lake. Science, 350(6267), 1493-1498. https://doi.org/10.1126/science.aac9927

Malinsky, Milan, Svardal, H., Tyers, A. M., Miska, E. A., Genner, M. J., Turner, G. F., \& Durbin, R. (2018). Whole-genome sequences of Malawi cichlids reveal multiple radiations interconnected by gene flow. Nature Ecology \& Evolution, 2(12), 1940-1955. https://doi.org/10.1038/s41559-018-0717-x

Markert, J. A., \& Arnegard, M. E. (2007). Size-dependent use of territorial space by a rock-dwelling cichlid fish. Oecologia, 154(3), 611-621. 
Matsuda, M., \& Sakaizumi, M. (2016). Evolution of the sex-determining gene in the teleostean genus Oryzias. General and Comparative Endocrinology, 239, 80-88. https://doi.org/10.1016/j.ygcen.2015.10.004

McKenna, A., Hanna, M., Banks, E., Sivachenko, A., Cibulskis, K., Kernytsky, A., Garimella, K., Altshuler, D., Gabriel, S., Daly, M., \& DePristo, M. A. (2010). The Genome Analysis Toolkit: A MapReduce framework for analyzing next-generation DNA sequencing data. Genome Research, 20(9), 1297-1303. https://doi.org/10.1101/gr.107524.110

Myosho, T., Otake, H., Masuyama, H., Matsuda, M., Kuroki, Y., Fujiyama, A., Naruse, K., Hamaguchi, S., \& Sakaizumi, M. (2012). Tracing the Emergence of a Novel Sex-Determining Gene in Medaka, Oryzias luzonensis. Genetics, 191(1), 163-170. https://doi.org/10.1534/genetics.111.137497

Nelson, C.M. (1995). Male size, spawning pit size and female mate choice in a lekking cichlid fish. Animal Behaviour, 50(6), 1587-1599.

$$
\text { https://doi.org/10.1016/0003-3472(95)80013-1 }
$$

Parnell, N. F., \& Streelman, J. T. (2013). Genetic interactions controlling sex and color establish the potential for sexual conflict in Lake Malawi cichlid fishes. Heredity, 110(3), 239-246. https://doi.org/10.1038/hdy.2012.73

Pennell, M. W., Mank, J. E., \& Peichel, C. L. (2018). Transitions in sex determination and sex chromosomes across vertebrate species. Molecular Ecology, 27(19), 3950-3963. https://doi.org/10.1111/mec.14540

Peterson, E. N., Cline, M. E., Moore, E. C., Roberts, N. B., \& Roberts, R. B. (2017). Genetic sex determination in Astatotilapia calliptera, a prototype species for the Lake Malawi cichlid radiation. Die Naturwissenschaften, 104(5-6), 41. https://doi.org/10.1007/s00114-017-1462-8

Poplin, R., Ruano-Rubio, V., DePristo, M. A., Fennell, T. J., Carneiro, M. O., Van der Auwera, G. A., Kling, D. E., Gauthier, L. D., Levy-Moonshine, A., Roazen, D., Shakir, K., Thibault, J., Chandran, S., Whelan, C., Lek, M., Gabriel, S., Daly, M. J., Neale, B., MacArthur, D. G., \& Banks, E. (2017). Scaling accurate genetic variant discovery to tens of thousands of samples [Preprint]. bioRxiv. https://doi.org/10.1101/201178

Purcell, S. (2014). PLINK 1.9. http://pngu.mgh.harvard.edu/purcell/plink/

Purcell, S., Neale, B., Todd-Brown, K., Thomas, L., Ferreira, M. A. R., Bender, D., Maller, J., Sklar, P., de Bakker, P. I. W., Daly, M. J., \& Sham, P. C. (2007). PLINK: A tool set for whole-genome association and population-based linkage analyses. American Journal of 
R Core Team. (2020). R: A Language and Environment for Statistical Computing. R Foundation for Statistical Computing. https://www.R-project.org/

Rhie, A., McCarthy, S. A., Fedrigo, O., Damas, J., Formenti, G., Koren, S., Uliano-Silva, M., Chow, W., Fungtammasan, A., Kim, J., Lee, C., Ko, B. J., Chaisson, M., Gedman, G. L., Cantin, L. J., Thibaud-Nissen, F., Haggerty, L., Bista, I., Smith, M., ... Jarvis, E. D. (2021). Towards complete and error-free genome assemblies of all vertebrate species. Nature, 592(7856), 737-746. https://doi.org/10.1038/s41586-021-03451-0

Roberts, R. B., Ser, J. R., \& Kocher, T. D. (2009). Sexual Conflict Resolved by Invasion of a Novel Sex Determiner in Lake Malawi Cichlid Fishes. Science, 326(5955), 998-1001. https://doi.org/10.1126/science.1174705

Robinson, J. T., Thorvaldsdóttir, H., Winckler, W., Guttman, M., Lander, E. S., Getz, G., \& Mesirov, J. P. (2011). Integrative genomics viewer. Nature Biotechnology, 29(1), 24-26. https://doi.org/10.1038/nbt.1754

Robinson, M. D., McCarthy, D. J., \& Smyth, G. K. (2010). edgeR: A Bioconductor package for differential expression analysis of digital gene expression data. Bioinformatics, 26(1), 139-140. https://doi.org/10.1093/bioinformatics/btp616

Ronco, F., Büscher, H. H., Indermaur, A., \& Salzburger, W. (2020). The taxonomic diversity of the cichlid fish fauna of ancient Lake Tanganyika, East Africa. Journal of Great Lakes Research, 46(5), 1067-1078. https://doi.org/10.1016/j.jglr.2019.05.009

Saunders, P. A., Neuenschwander, S., \& Perrin, N. (2018). Sex chromosome turnovers and genetic drift: A simulation study. Journal of Evolutionary Biology, 31(9), 1413-1419. https://doi.org/10.1111/jeb.13336

Schartl, M., Schories, S., Wakamatsu, Y., Nagao, Y., Hashimoto, H., Bertin, C., Mourot, B., Schmidt, C., Wilhelm, D., Centanin, L., Guiguen, Y., \& Herpin, A. (2018). Sox5 is involved in germ-cell regulation and sex determination in medaka following co-option of nested transposable elements. BMC Biology, 16(1), 16. https://doi.org/10.1186/s12915-018-0485-8

Sefc, K. M. (2011). Mating and Parental Care in Lake Tanganyika's Cichlids. International Journal of Evolutionary Biology, 2011, 1-20. https://doi.org/10.4061/2011/470875

Ser, J. R., Roberts, R. B., \& Kocher, T. D. (2010). Multiple interacting loci control sex determination in Lake Malawi cichlid fish. Evolution, 64(2), 486-501. https://doi.org/10.1111/j.1558-5646.2009.00871.x

Theis, A., Bosia, T., Roth, T., Salzburger, W., \& Egger, B. (2015). Egg-spot pattern and body 
1000 Zhou, X., \& Stephens, M. (2012). Genome-wide efficient mixed-model analysis for association 1001 1002 Zhu, Y., Meng, L., Xu, W., Cui, Z., Zhang, N., Guo, H., Wang, N., Shao, C., \& Chen, S. (2018). 1003

size asymmetries influence male aggression in haplochromine cichlid fishes. Behavioral Ecology, 26(6), 1512-1519. https://doi.org/10.1093/beheco/arv104

Turner, G. F., \& Huntingford, F. A. (1986). A problem for game theory analysis: Assessment and intention in male mouthbrooder contests. Animal Behaviour, 34(4), 961-970. https://doi.org/10.1016/S0003-3472(86)80155-5

Turner, G., Ngatunga, B. P., \& Genner, M. J. (2019). The Natural History of the Satellite Lakes of Lake Malawi [Preprint]. EcoEvoRxiv. https://doi.org/10.32942/osf.io/sehdq

Van der Auwera, G., \& O'Connor, B. (2020). Genomics in the Cloud: Using Docker, GATK, and WDL in Terra (1st ed.). O'Reilly Media.

van Doorn, G. S., \& Kirkpatrick, M. (2007). Turnover of sex chromosomes induced by sexual conflict. Nature, 449(7164), 909-912. https://doi.org/10.1038/nature06178

van Doorn, G. S., \& Kirkpatrick, M. (2010). Transitions between male and female heterogamety caused by sex-antagonistic selection. Genetics, 186(2), 629-645. https://doi.org/10.1534/genetics.110.118596

Vicoso, B. (2019). Molecular and evolutionary dynamics of animal sex-chromosome turnover. Nature Ecology \& Evolution, 3(12), 1632-1641. https://doi.org/10.1038/s41559-019-1050-8

Vieira, F. G., Lassalle, F., Korneliussen, T. S., \& Fumagalli, M. (2016). Improving the estimation of genetic distances from Next-Generation Sequencing data: Genetic Distances from NGS Data. Biological Journal of the Linnean Society, 117(1), 139-149. https://doi.org/10.1111/bij.12511

Werren, J. H., \& Beukeboom, L. W. (1998). Sex determination, sex ratios, and genetic conflict. Annual Review of Ecology and Systematics, 29(1), 233-261. https://doi.org/10.1146/annurev.ecolsys.29.1.233

Williamson, D., Jackson, M. J., Banerjee, S. K., Marvin, J., Merdaci, O., Thouveny, N., Decobert, M., Gibert-Massault, E., Massault, M., Mazaudier, D., \& Taieb, M. (1999). Magnetic signatures of hydrological change in a tropical maar-lake (Lake Massoko, Tanzania): Preliminary results. Physics and Chemistry of the Earth, Part A: Solid Earth and Geodesy, 24(9), 799-803. https://doi.org/10.1016/S1464-1895(99)00117-9 studies. Nature Genetics, 44(7), 821-824. https://doi.org/10.1038/ng.2310 The autosomal Gsdf gene plays a role in male gonad development in Chinese tongue sole (Cynoglossus semilaevis). Scientific Reports, 8(1), 17716. 
bioRxiv preprint doi: https://doi.org/10.1101/2021.08.05.455235; this version posted October 5, 2021. The copyright holder for this preprint

(which was not certified by peer review) is the author/funder, who has granted bioRxiv a license to display the preprint in perpetuity. It is made available under aCC-BY-NC-ND 4.0 International license.

1005

https://doi.org/10.1038/s41598-018-35553-7

1006 Supplementary Figures \& Tables 
bioRxiv preprint doi: https://doi org/10.1101/2021.08.05.455235. this version posted October 5,2021 . The copyright holder for this preprint (which was not certified by peer review) is the author/funder, who has granted bioRxiv a license to display the preprint in perpetuity. It is made available under aCC-BY-NC-ND 4.0 International license.

(a)
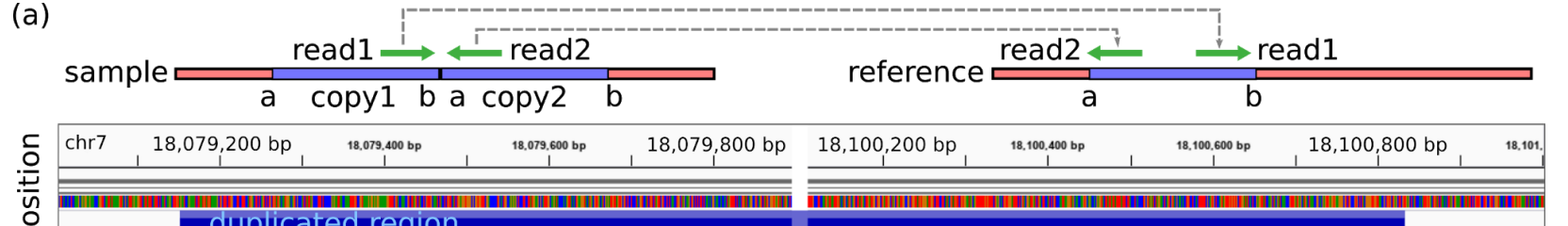

$\stackrel{0}{\circ}$

duplicated region -

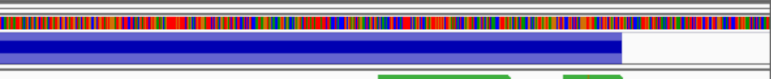

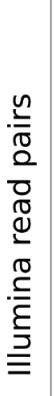

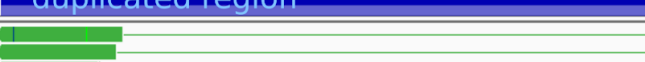

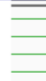
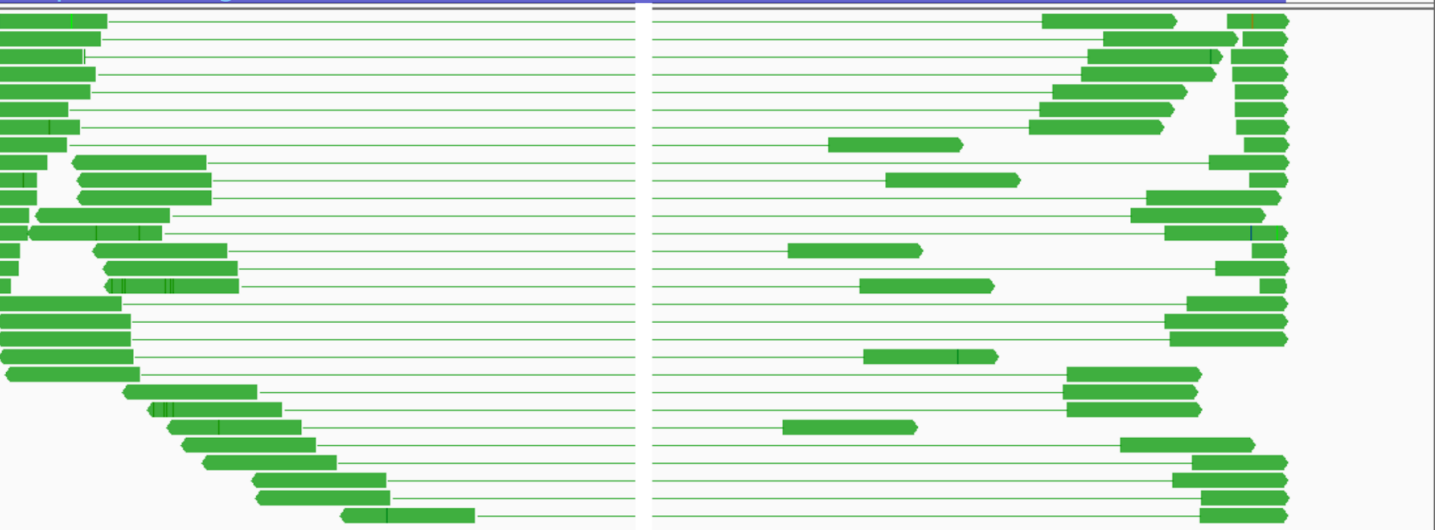

(b)

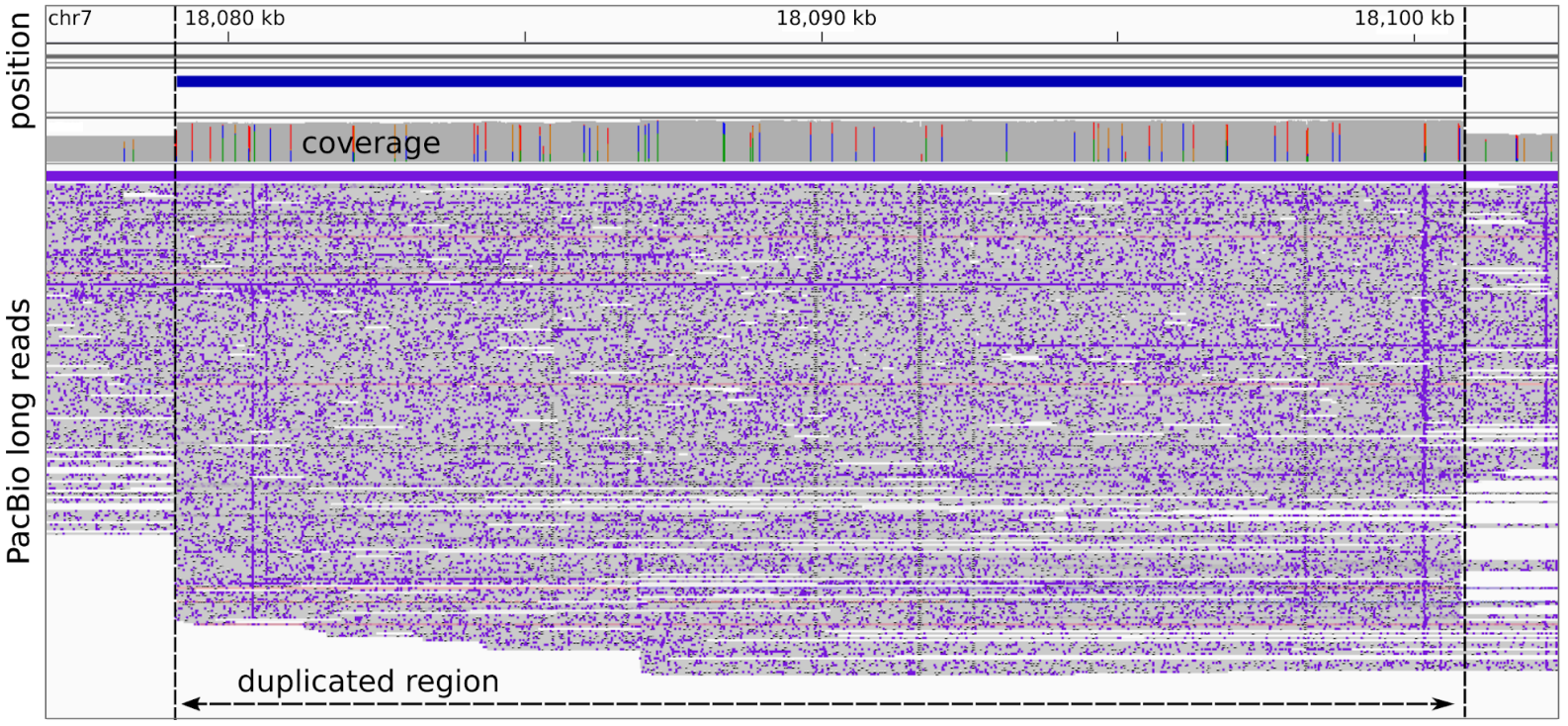

(c)

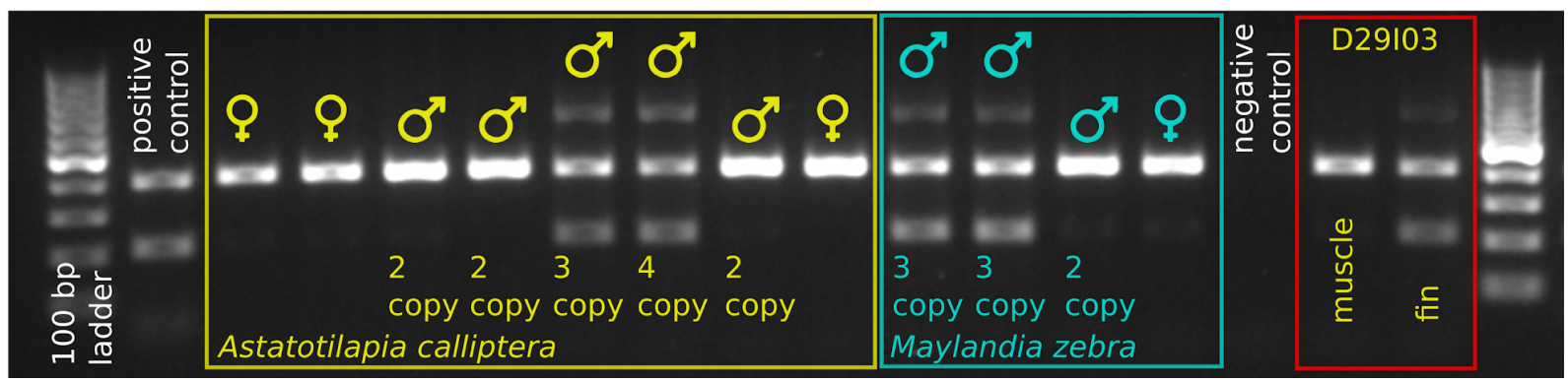

1007 Figure S1: Characterization of the gsdf duplication. (a) Short Illumina reads from four

1008 Masoko male A. calliptera called homozygous for the gsdf duplication based on relative 
1009 sequencing depth that is approximately 2x higher than in $\sim 38 \mathrm{~kb}$ of non-duplicated flanking 1010 sequence. The mapping orientation of all read pairs to the fAstCal1.2 reference is consistent 1011 with a tandem duplication as shown in the schematic at the top. (b) PacBio reads from a male 1012 Tropheops 'mauve' mapped to the fAstCal1.2 reference. The sharp break in the alignment of 1013 some of the reads at the edges of the gsdf duplication (blue horizontal bar) in conjunction with 1014 elevated coverage signals that this individual is heterozygous for the same gsdf duplication 1015 identified in Masoko A. calliptera. (c) Agarose gel image of PCR products from primers 1016 designed to assay for the presence of the gsdf duplication. Based on this assay, individuals 1017 positive for the $g s d f$ duplication yield three distinct bands, whereas those negative for the 1018 duplication produce a single band. The assay was used to confirm the presence of the 1019 duplication in two male Maylandia zebra samples that were putative heterozygotes for gsdf-dup 1020 based on sequencing depth. Two separate tissues for Masoko A. calliptera sample D29I03 1021 produced different genotypes based on this PCR assay indicating a sampling error and resulted 1022 in this individual being omitted from all analyses.
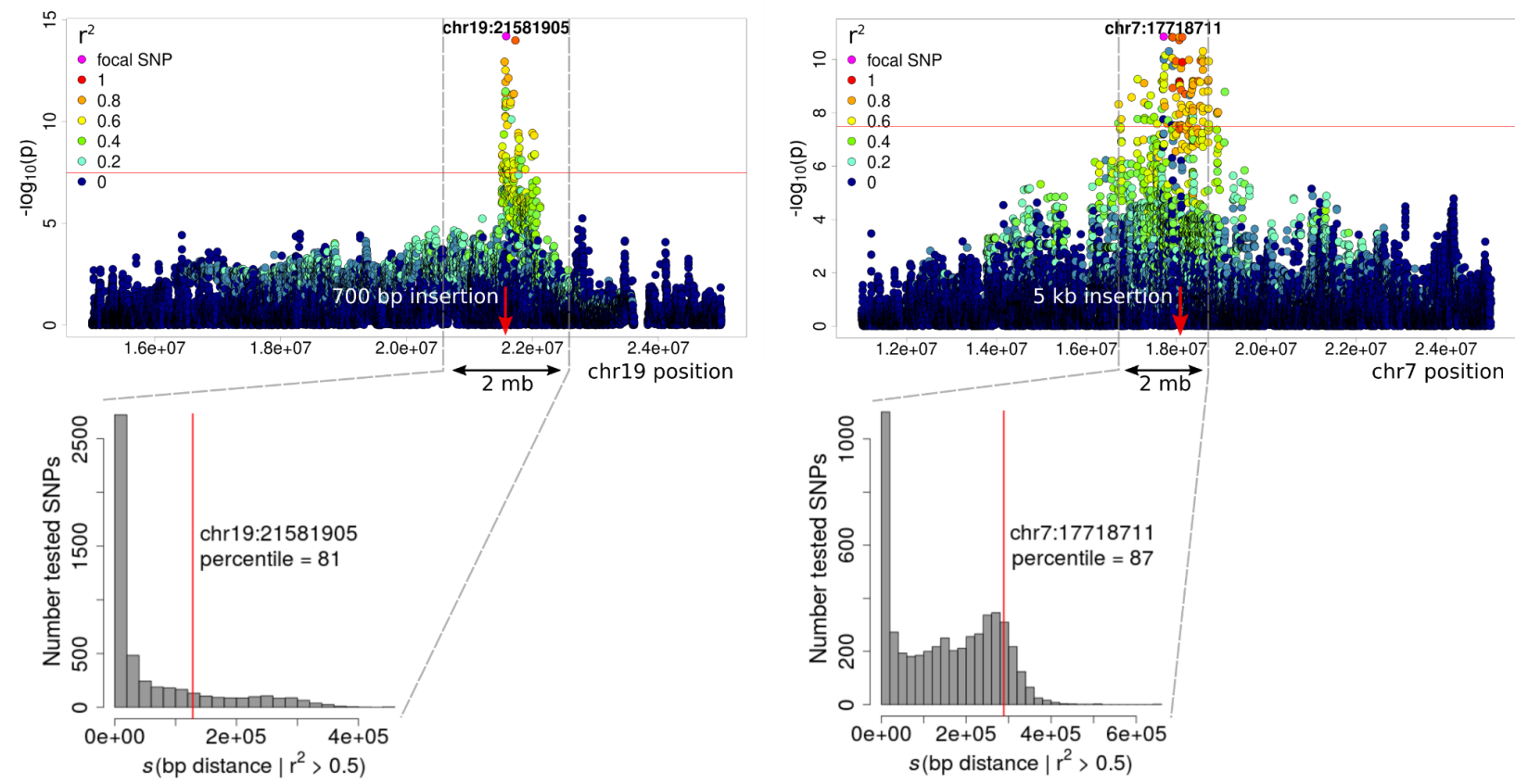

1023 Figure S2: Elevated linkage disequilibrium around the chr19-ins and chr7-ins loci. The top 1024 Manhattan plots are a regional view of the $p$-values for the likelihood ratio test from the GWAS 1025 for sex used to identify SNPs tagging chr19-ins (left) and chr7-ins (right). The positions of the 1026 insertions are denoted with red arrows. Elevated linkage disequilibrium (LD) between the SNP 1027 with the highest sex association in each GWAS and other surrounding SNPs extends far along 
1028 the respective chromosomes. This causes the variance in the pairwise physical distance among 1029 SNPs in high LD $\left(r^{2}>0.5\right)$ with the top GWAS SNPs to be higher than typically expected 1030 throughout the genome, consistent with recent positive selection. The histograms show where 1031 this variance for the top GWAS SNPs fall along the expected distributions for Masoko $A$.

1032 calliptera, which were generated by randomly sampling 5,000 SNPs across the genome with the 1033 same alternate allele frequencies as the GWAS SNPs. The variance among the pairwise 1034 distances between each sampled SNP and their surrounding high-LD SNPs were calculated in 1035 the same manner as for the GWAS SNPs.
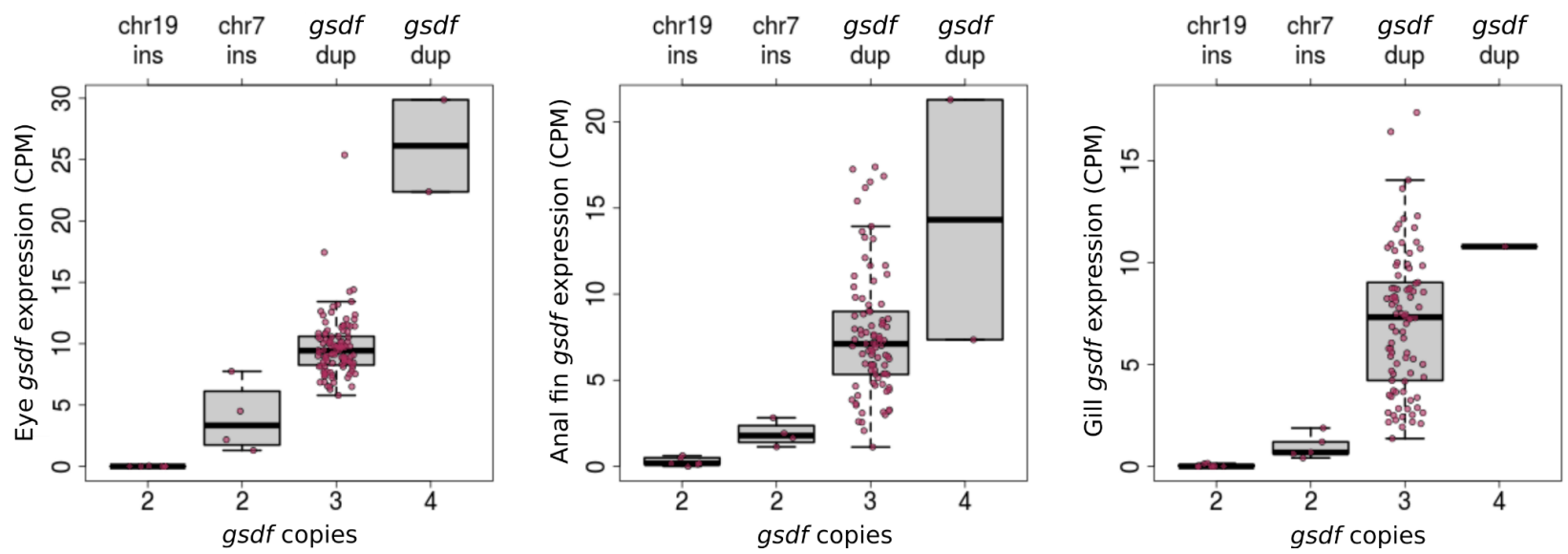

1036 Figure S3: Expression of $\mathbf{g s d f}$ in somatic tissues for males with different $\mathbf{Y}$ alleles. The

1037 gsdf-dup and chr7-ins alleles are defined by a tandem duplication of the gsdf gene and an

1038 insertion directly upstream of $g s d f$, respectively. Levels of $g s d f$ expression in eye, anal fin, and 1039 gill tissues from Masoko male $A$. calliptera demonstrate that males carrying putative $Y$ alleles 1040 generated through mutations involving gsdf express this gene more than other males. 


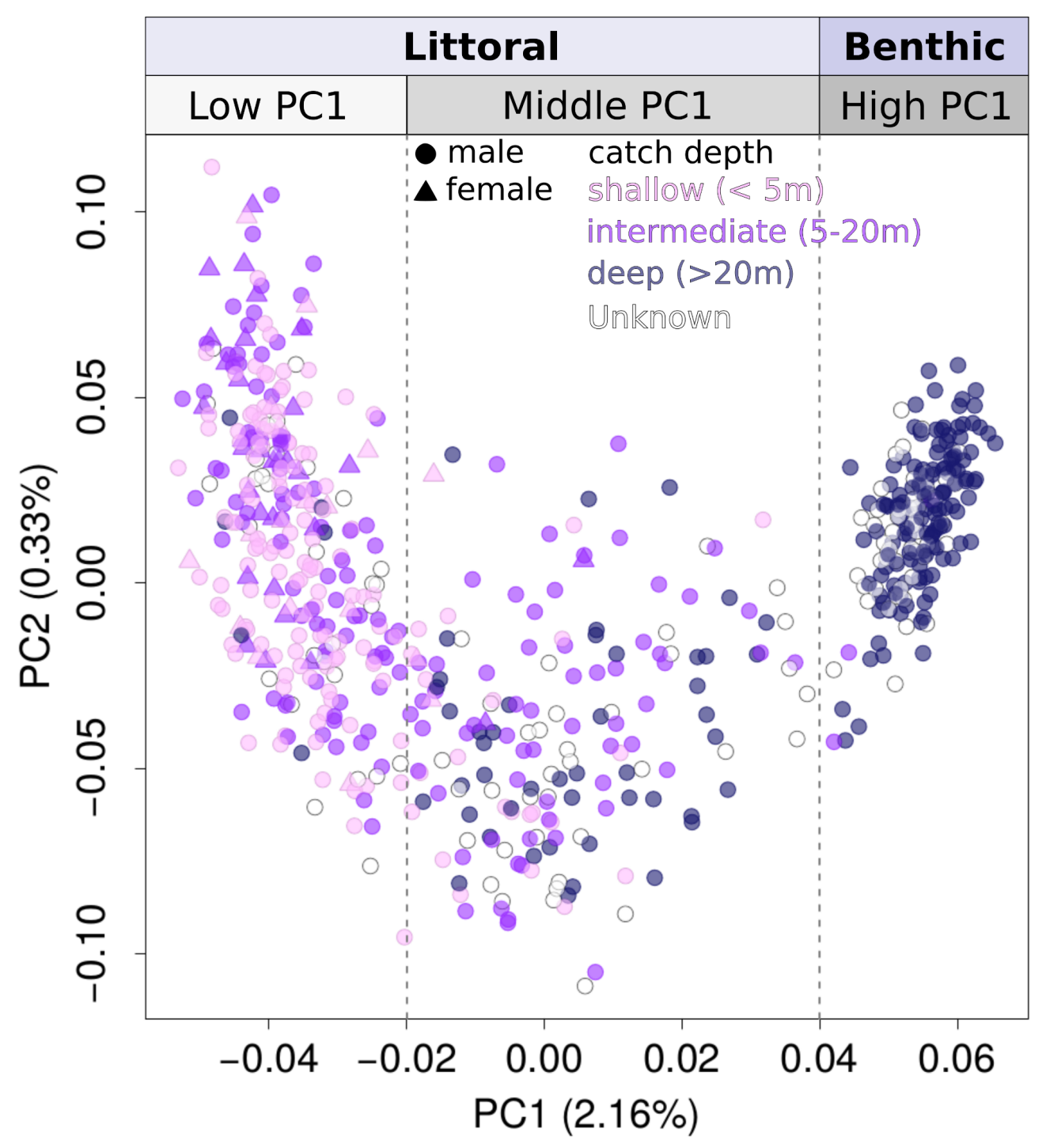

1041 Figure S4: Relationship between genetic variation and catch depth. Lake Masoko $A$.

1042 calliptera distributed along the first two components of a principal component analysis of

1043 genome-wide variation reveals strong philopatry of high PC1 fish for deep depths. This

1044 coincides with nearly all high PC1 individuals conforming to the benthic ecomorph. In contrast,

1045 fish below PC1 values of 0.04 are almost all of the littoral ecomorph and exhibit far less

1046 constrained habitat preference. Among littoral fish (PC1 < 0.04), the most admixed individuals in 1047 the middle of PC1 (-0.02 to 0.04) regularly occupy all depth bands, while low PC1 littorals (PC1 $1048<-0.02)$ remain mostly at depths above 20 metres, though occasionally they are found deep. 
(a)

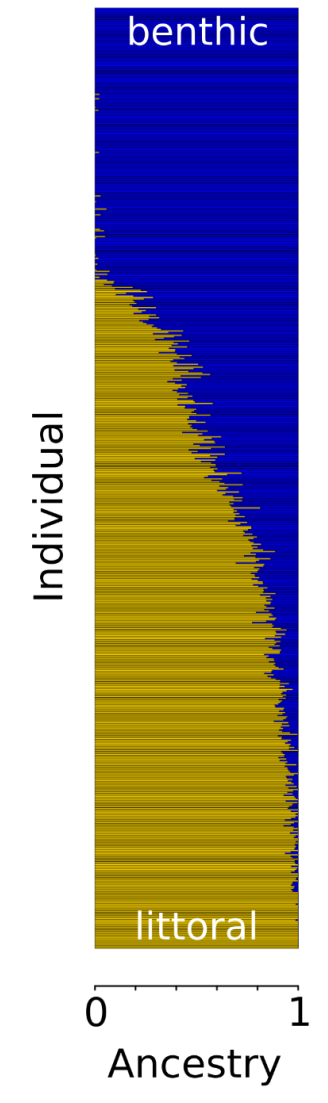

(b)

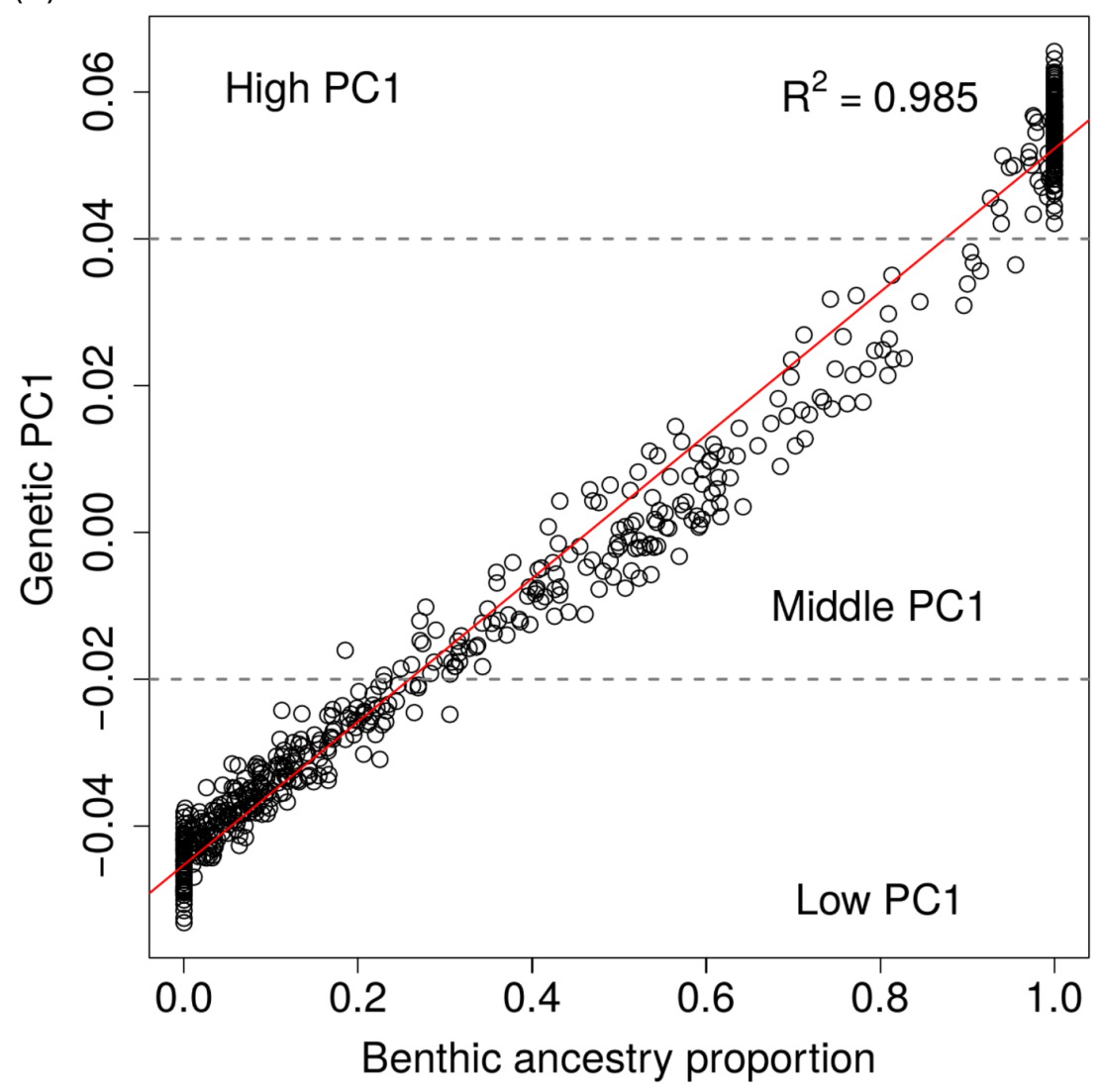

1049 Figure S5: Ancestry characterization of Masoko A. calliptera. (a) Genome-wide ancestry

1050 proportions for individuals inferred using the program ADMIXTURE and ordered by their genetic

1051 PC1 rank shows the genetic distinctiveness of the benthic (high PC1) subgroup, a subset of

1052 littorals having low amounts of benthic ancestry (low PC1), and a highly admixed group (middle

1053 PC1). (b) The genetic PC1 scores of Lake Masoko individuals regressed against their

1054 proportion of benthic ancestry shows that PC1 almost perfectly describes the genetic structure

1055 of the Lake Masoko population in terms of the continuum between genetically benthic and

1056 littoral ancestries. The fitted linear regression line is shown in red and the low, middle, and high

1057 PC1 classification cutoffs are depicted with dashed grey lines. 


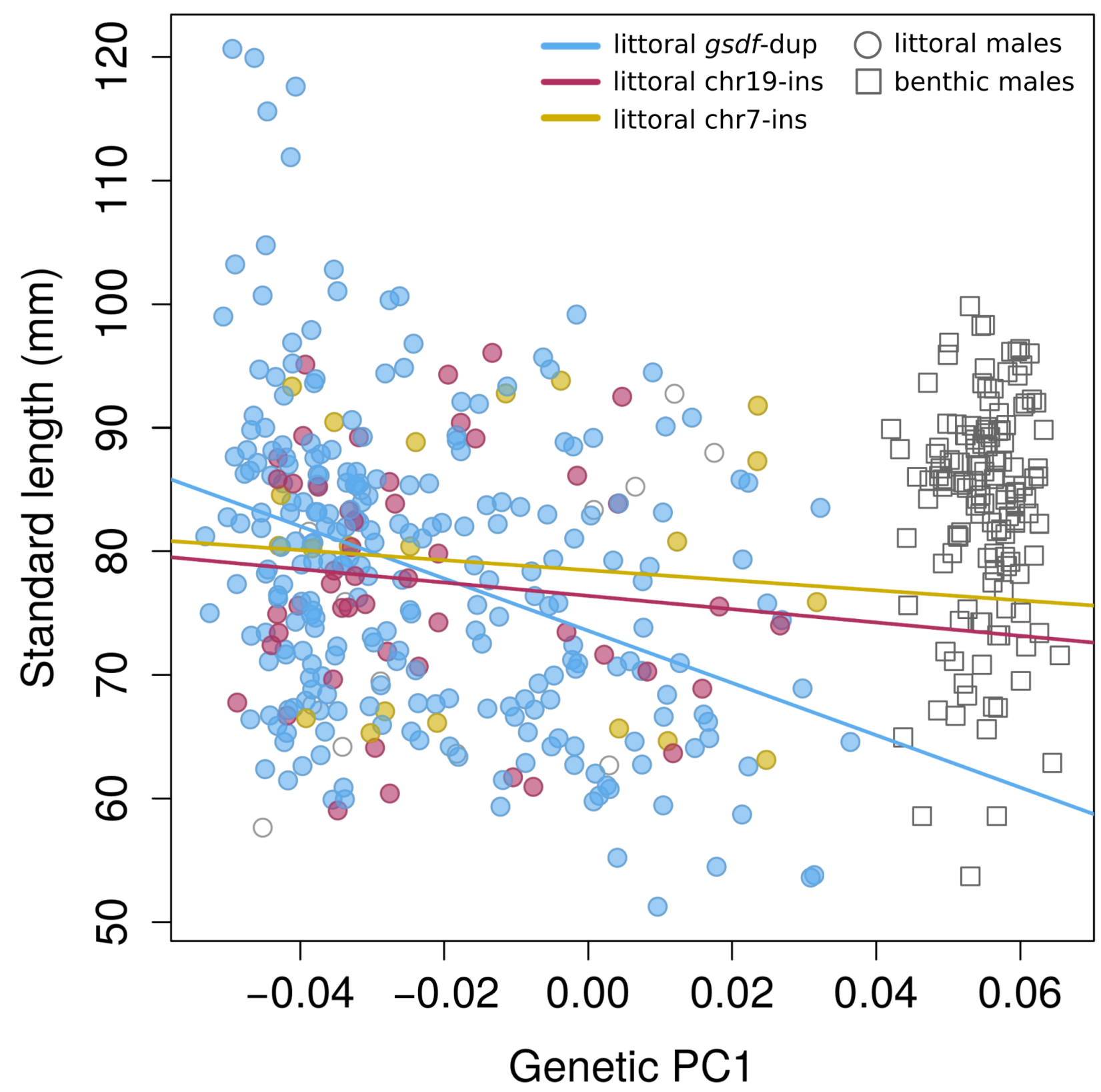

1058 Figure S6: Interaction between genetic background and Y allele in predicting male size.

1059 The standard lengths of male $A$. calliptera from Lake Masoko plotted against their position along 1060 PC1 of the principal component analysis of genome-wide variation shows a negative trend in 1061 the length among genetically littoral (PC1 < 0.04) males (circles) with increasing PC1 value. 1062 Linear regression models of length predicted by PC1 were fitted separately for littoral males 1063 heterozygous for either gsdf-dup, chr19-ins, or chr7-ins corresponding to the colours blue, red, 1064 and yellow, respectively. Littoral males carrying more than one $Y$ allele, homozygous for $Y$ 1065 alleles, or which did not have an identified Y, are represented by uncoloured circles and were 
1066 excluded from the regressions. Genetically benthic males, defined as fish with PC1 > 0.04, are 1067 plotted for comparative purposes as squares without any indication of their $Y$ genotype. The 1068 distinctly more negative slope of the regression line fit to gsdf-dup males compared to chr19-ins 1069 and chr7-ins males shows that length is predicted to decrease much more drastically with more 1070 benthic admixture among gsdf-dup males. This difference is so great that males using gsdf-dup 1071 are predicted to switch from being longer than males using other $Y$ alleles to actually being 1072 shorter above PC1 values of -0.02 . 


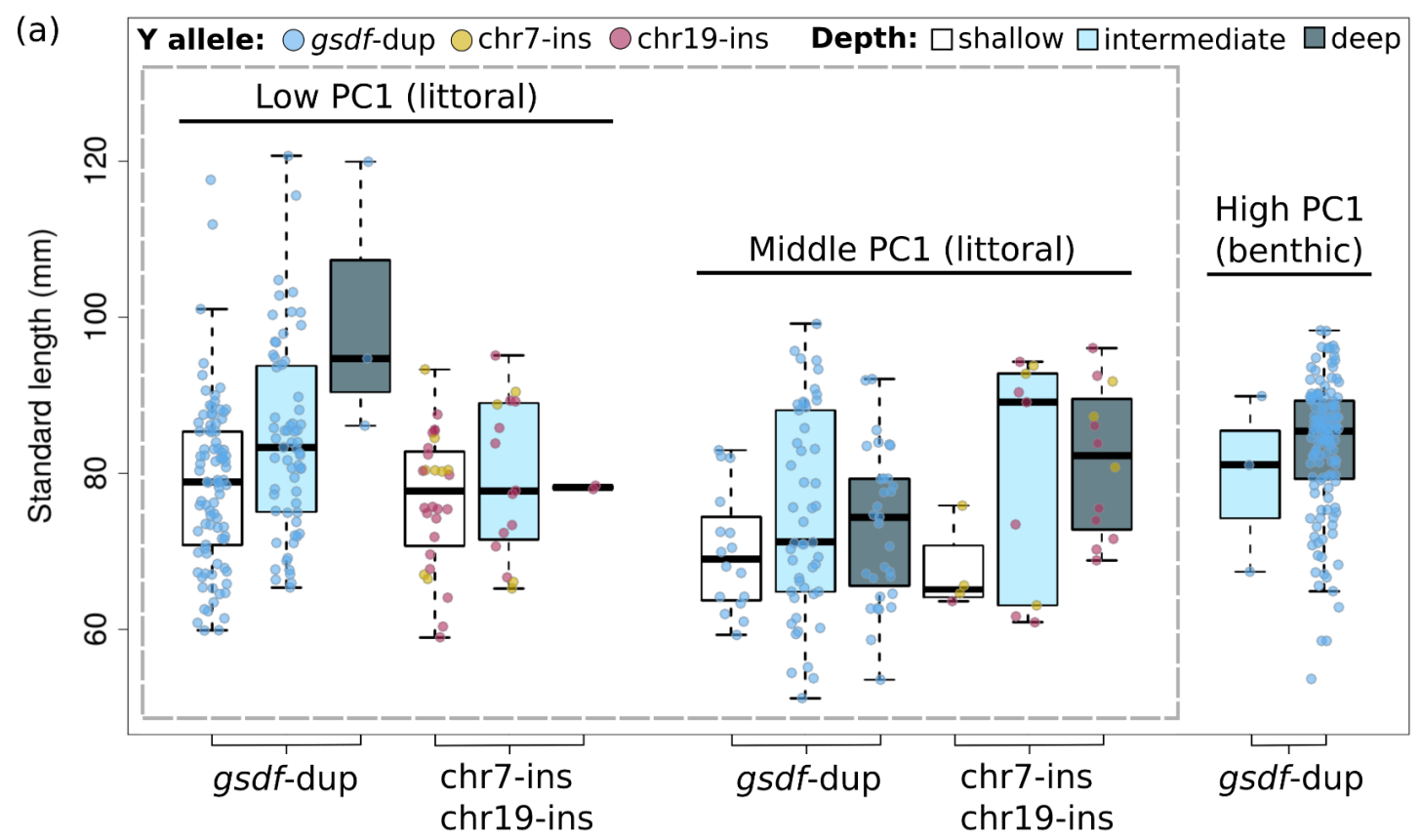

Sex system

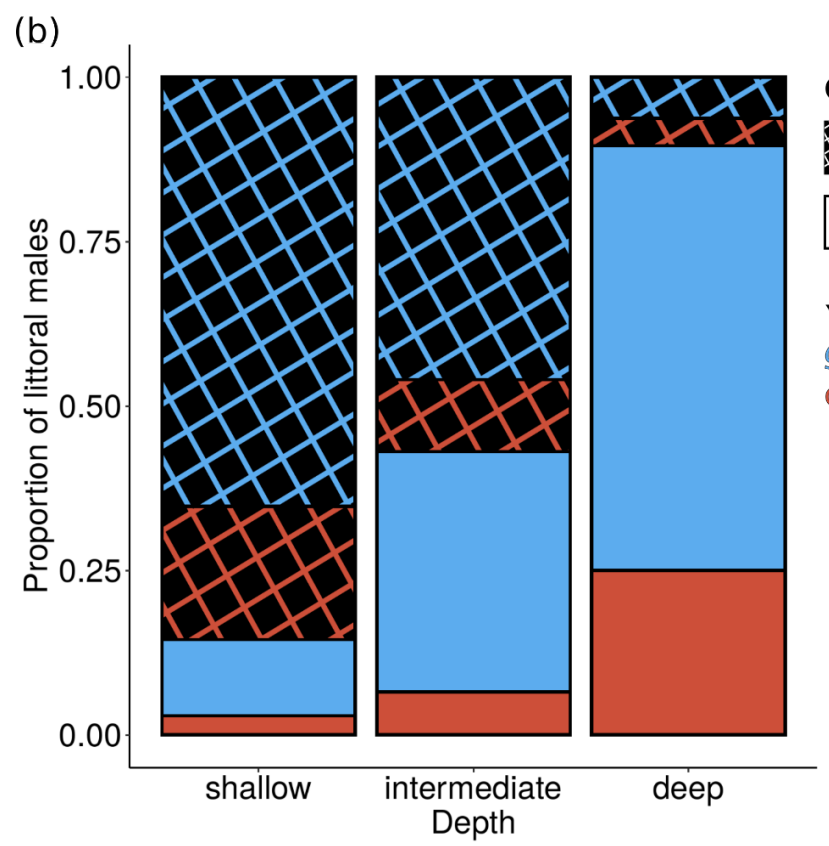

Genomic background

戈 Low PC1

Middle PC1

Y allele

gsdf-dup

chr7-ins or chr19-ins

\section{Figure S7: Male sizes and frequencies according to $Y$ allele, genetic PC1, and catch}

1074 depth. (a) Standard length comparisons across different PC1 genetic backgrounds and catch

1075 depths of Lake Masoko A. calliptera males heterozygous for only one of the $Y$ alleles shows an

1076 interaction between $\mathrm{Y}$ allele, catch depth, and PC1 background in predicting size. Among the

1077 genetically littoral males (within the dashed grey box) those carrying gsdf-dup are smaller on

1078 middle PC1 versus low PC1 backgrounds regardless of what depth they are found at. In 
1079 contrast, among males using the other Y alleles only middle PC1 males found in shallow waters

1080 are smaller than the low PC1 males, while at deeper depths their size remains constant across

1081 genetic backgrounds and may even show a subtle tendency to be larger with middle PC1

1082 benthic ancestry. (b) A comparison of the proportion of littoral males characterized by different

1083 genetic PC1 backgrounds and $\mathrm{Y}$ alleles at different catch depths shows that the proportion of

1084 males with middle PC1 ancestry increases with depth. However, within PC1 backgrounds, the

1085 fraction of males using the different $Y$ alleles remains relatively stable across depths. Overall,

1086 gsdf-dup males dominate at all depths.

1087 Tables S1 to S7 can be found in the attached Excel file:

1088 supplementary_tables_differential_use_of_multiple_genetic_sex_determination_systems_in_div

1089 ergent_ecomorphs_of_an_African_crater_lake_cichlid.xls. For convenience the table legends

1090 are given below, and we also copy below the contents of tables S3 and S7, which are short.

1091 Table S1: Lake Masoko Astatotilapia calliptera samples Genetic, phenotypic, collection, and

1092 data availability information for all Lake Masoko A. calliptera samples. RNAseq expression

1093 levels for gsdf are reported in counts per million reads mapped (CPM). Sample accessions are

1094 provided for whole-genome (WGS) and RNAseq sequence data deposited into the European

1095 Nucleotide Archive. Missing values are coded as "NA".

1096 Table S2: GWAS multilocus sex determination genotype frequencies Counts of Masoko $A$.

1097 calliptera individuals, stratified by sex and PC1 genetic background, for all observed

1098 combinations of $g s d f$ copy number and genotypes at the most strongly associated SNPs in the

1099 serial GWAS for sex. 0 = reference allele, $1=$ insertion allele, .l. = missing genotype.

1100 Table S3: Average sizes of Masoko males The mean standard length of Masoko A. calliptera

1101 males heterozygous for one type of $Y$ allele stratified by PC1 genetic background and catch

1102 depth.

\begin{tabular}{|c|c|c|}
\hline \multicolumn{3}{|c|}{ Lake-wide mean length (mm) } \\
\hline Y allele & Low PC1 & Middle PC1 \\
\hline gsdf-dup & 81.34 & 73.55 \\
\hline
\end{tabular}




\begin{tabular}{|c|c|c|}
\hline chr7-ins or chr19-ins & 77.68 & 78.73 \\
\hline \multicolumn{3}{|c|}{ Shallow $(<5 \mathrm{~m})$ mean length $(\mathrm{mm})$} \\
\hline $\mathrm{Y}$ allele & Low PC1 & Middle PC1 \\
\hline gsdf-dup & 78.55 & 69.91 \\
\hline chr7-ins or chr19-ins & 76.67 & 67.46 \\
\hline \multicolumn{3}{|c|}{ Intermediate $(5-20 \mathrm{~m})$ mean length $(\mathrm{mm})$} \\
\hline Y allele & Low PC1 & Middle PC1 \\
\hline gsdf-dup & 84.41 & 74.87 \\
\hline chr7-ins or chr19-ins & 79.50 & 79.96 \\
\hline \multicolumn{3}{|c|}{ Deep $(>20 \mathrm{~m})$ mean length $(\mathrm{mm})$} \\
\hline Y allele & Low PC1 & Middle PC1 \\
\hline gsdf-dup & 100.26 & 73.33 \\
\hline chr7-ins or chr19-ins & 78.22 & 81.56 \\
\hline
\end{tabular}

1103 Table S4: Littoral male frequencies according to genetic type and catch depth Counts of

1104 Lake Masoko A. calliptera littoral males heterozygous for one type of $Y$ allele stratified by 1105 genetic PC1 background and depth at which they were caught.

1106 Table S5: Sex loci genotype calls for Lake Malawi cichlid radiation species The number of 1107 gsdf copies and genotype (GT) calls for chr19-ins and chr7-ins ( $0=$ reference allele, $1=$ 
1108 insertion allele, ./. = missing genotype) for individuals of different species belonging to the Lake

1109 Malawi haplochromine cichlid radiation. The AC values indicate the number of "<reference

1110 allele>,<insertion allele>" sequencing reads observed for an individual. Missing values are

1111 coded as "NA".

\section{Table S6: Frequency of chr7-ins in non-calliptera species from the Lake Malawi}

1113 haplochromine radiation Counts of individuals from all species apart from Astatotilapia

1114 calliptera in which chr7-ins was found, stratified by gsdf copy number and chr7-ins genotype.

1115 Multilocus genotype calls are defined as <number of $g$ sdf copies $>\mid<$ number of chr7-ins alleles $>$ :

1116 for example, "3/1" denotes an individual possessing three gsdf copies and who is heterozygous

1117 for the insertion allele at the chr7-ins locus. Genotype class cells with non-zero counts are

1118 highlighted for readability.

1119 Table S7: PCR primers for the detection of gsdf-dup All samples should undergo

1120 amplification for the 402 bp control fragment, whereas only samples positive for the gsdf

1121 duplication should show equally strong amplification for the $207 \mathrm{bp}$ fragment (and an additional

$1122614 \mathrm{bp}$ fragment which is not present when each primer pair is run in individual reactions).

\begin{tabular}{|c|c|c|c|c|c|}
\hline primer & sequence & $\begin{array}{c}\text { Tm } \\
\left({ }^{\circ} \mathrm{C}\right)\end{array}$ & $\begin{array}{l}\% G C \\
\text { primer }\end{array}$ & $\begin{array}{c}\text { amplicon } \\
\text { size (bp) }\end{array}$ \\
\hline dup_fwd & TGTCGCGTCATAACGAGGAG & 59.9 & 55 & dup_rev & 207 \\
\hline dup_rev & AGCTGATCTGGTCCCTCACT & 60.0 & 55 & dup_fwd & \\
\hline \multirow{2}{*}{ control_fwd } & GCTGCCCACCTCGTAGTAAT & 59.5 & 55 & control_rev & \\
\hline control_rev & GCACGAGTGGGAACCAGTAA & 60.0 & 55 & control_fwd & \\
\hline
\end{tabular}


bioRxiv preprint doi: https://doi.org/10.1101/2021.08.05.455235; this version posted October 5, 2021. The copyright holder for this preprint (which was not certified by peer review) is the author/funder, who has granted bioRxiv a license to display the preprint in perpetuity. It is made available under aCC-BY-NC-ND 4.0 International license.

\begin{tabular}{|c|c|c|c|c|c|}
\hline dup_fwd & & & & control_rev & 614 \\
\hline
\end{tabular}

